\title{
A Framework to Channel Feedback and Reconstruction using Matrix Completion for Massive MIMO Systems
}

\author{
Samuel T. Valduga, André L. F. de Almeida, Carlos Filipe M. Silva, Igor M. Guerreiro, and Daniel C. Araújo
}

\begin{abstract}
In this paper, we are interested in the problem of channel feedback and reconstruction in frequency division duplexing (FDD)-based massive multiple-input-multiple-output (MIMO) systems. We propose a general framework that allows reducing the overhead significantly in the uplink feedback control channel when assuming massive antenna arrays at both ends of the wireless link. The fundamental idea of the proposed method is to explore the low-rank structure of the channel for its accurate reconstruction at the transmitter side (Tx) with very few uplink feedback information, using matrix completion techniques. The proposed framework consists of two stages. First, upon reception of downlink pilots, the receiver side $(\mathrm{Rx})$ undersamples either the received pilot data matrix or the estimated channel matrix and feeds back only a fraction of their entries to the $T x$, throwing away the remaining ones. Then, under the assumption of reduced scattering propagation, the $T x$ capitalizes on matrix completion to recover either the downlink pilots or to directly reconstruct the downlink channel. We consider two application examples: i) backhauling communication and ii) a multi-user scenario with perfect and imperfect instantaneous channel knowledge. Due to data/channel undersampling, energy consumption at a receiver can be reduced during the uplink feedback transmission. Simulation results show that, compared to the conventional full-feedback approach, which requires feedback of the entire data/channel matrix, the proposed solution can decrease the feedback load in more than $90 \%$ for low-rank channels, while providing good channel estimation accuracy, bit error rate (BER) and goodput assuming maximum ratio transmission (MRT) precoding.
\end{abstract}

Index Terms-5G, quantized feedback, matrix completion, backhaul, multi-user, FDD, BER, MRT, channel estimation.

\section{INTRODUCTION}

$\mathbf{M}$ ASSIVE multiple-input-multiple-output (MIMO) is considered as one of the key technologies for the fifth generation $(5 \mathrm{G})$ of wireless communication systems due to its potential to achieve high data rates and its robustness against interference, fading, hardware imperfections and antenna element failure [1]. In his seminal paper, Marzetta [2]

The Associate Editor coordinating the review of this manuscript approving it for publication was Prof. José Cândido Silveira Santos Filho.

Samuel T. Valduga is with the Federal University of Santa Maria (UFSM), Santa Maria, RS, 97105-900, Brazil (e-mail: samuel.valduga@ufsm.br).

André L. F. de Almeida, Carlos Filipe M. Silva, Igor M. Guerreiro, and Daniel C. Araújo are with the Federal University of Ceará (UFC) Campus do Pici, s/n, CP 600560455-970, Fortaleza, CE, Brazil (e-mail: \{andre,cfms,igor,araujo\}@gtel.ufc.br)

This work was realized with partial support of CAPES (Coordenação de Aperfeiçoamento de Pessoal de Nível Superior, Brazil) and Innovation Center, Ericsson Telecomunicações S/A. Igor M. Guerreiro and Daniel C. Araújo would like to acknowledge $\mathrm{CNPq}$ for its financial support (process $\mathrm{nr}$. 151004/2017-0 and 167855/2017-4, respectively

Digital Object Identifier: 10.14209/jcis.2018.9 has shown that, when the number of antennas grows very large, the effect of additive noise diminishes, as well as the required transmitted energy per bit. Spatial focusing of energy into eversmaller regions of space potentially brings huge improvements in throughput and radiated energy efficiency. Other benefits also could include the extensive use of inexpensive low-power components, low latency communication, simplification of the medium access control (MAC) layer, and robustness to intentional jamming [1].

To fully utilize the benefits of such a promising technology, an accurate knowledge of the channel state information (CSI) at the base station (BS) is essential to apply linear precoders such as a simple maximum ratio transmission (MRT) or a zero forcing beamforming (ZFBF). In time division-duplexing (TDD) operation mode, the CSI can be obtained at the transmitter by exploiting channel reciprocity using uplink pilots. On the other hand, frequency division duplexing (FDD) is generally considered to be more effective under symmetric traffic and delay-sensitive applications due to small latency, continuous channel estimation, and backward compatibility. Moreover, FDD is employed in most existing wireless systems. Consequently, it is important to identify and develop solutions for potential issues arising on FDD-based massive MIMO technique.

Henceforth, we focus on FDD operation. In this context, one well-known problem is that the channel feedback overhead grows linearly with the number of antennas [3]-[6]. Then, for practical feedback channels with limited transmission rate, the overhead to obtain full CSI becomes prohibitively large due to the massive number of antenna elements. Thus, relying on CSI to design the downlink transmission is a bottleneck in FDD systems.

Solutions for reducing the amount of data to be sent via a limited feedback channel usually resort to compressed sensing (CS) techniques. These solutions consider that the channel matrices admit a sparse representation, for instance, because of the shared and limited local scattering. Therefore, due to the sparse channel structure, CS techniques can reduce the training sequence and feedback overheads [7]-[11]. In [7], a scheme to exploit the hidden joint sparse structure of channel matrices via CS has been proposed. In [8], an adaptive CSbased feedback scheme was proposed, where the feedback structure can be dynamically configured based on channel conditions, while [9] presents and discusses the use of sparsityinspired CSI acquisition techniques for massive MIMO, as well as the underlying mathematical theory in FDD and TDD 
modes. In [10], based on the spatial correlation and channel conditions, the authors suggest two compression methods for channel feedback to reduce the feedback overhead. A hybrid limited feedback design is proposed for massive MIMO in [11]. They consider quantized and codebook based feedbacks.

Solutions based on CS, however, rely on an adequate choice of a basis that provides a sparse representation. If such a basis fails, it no longer represents the channel and its spatial characteristics properly [10]. Additionally, a proper basis may need to be sent to the receiver, which increases the signaling overhead in this case. Differently, the authors in [12] proposed a channel estimation algorithm based on matrix completion (MC) technique, assuming a switch-based mmWave massive MIMO scenario. In general, MC is a technique that can be used to complete a matrix with missing elements. Comparing to CS technique, the authors showed that MC can achieve a nearoptimal spectral efficiency with significantly lower complexity, since it does not require any proper basis, and it is immune to array response mismatch, as well.

Another solution is to exploit spatial correlation information. For instance, [13] proposes to design grouping patterns, taking advantage of the spatial correlation mapping of multiple antenna elements to a single representative value, using predesigned patterns therein referred to as antenna group beamforming (AGB). However, full CSI is not available at the transmitter. Instead, the proposed scheme uses the pattern index to select the antenna group and the codeword index for transmit beamforming, making such a scheme limited. Another solution is presented in [14], which consists of a feedback scheme based on channel vector quantization and beamforming. The authors propose codebooks and user equipment (UE) selection for scheduling based on reliability information, channel quality indicator, channel direction indicator and rate approximation. However, this solution does not provide CSI to the transmitter.

The idea of applying a completion technique in MIMO communication appeared in [15] to obtain direction of arrival (DOA) for colocated MIMO radars. The solution consists of either performing a matched filtering with a small number of randomly selected dictionary waveforms or undersampling the received signal at random sampling instants and forwarding the results to a fusion center. From the received samples and the sampling scheme, the fusion center applies a matrix completion technique to estimate the full matrix. In [16], the authors proposed a solution to provide the CSI to the transmitter side (Tx) for FDD massive MIMO systems. The proposal is to apply an algorithm based on matrix completion concepts. They consider that all scheduled UEs directly feed the full received pilots back to the Tx, which applies a lowrank approximation for CSI recovery. Therein, the authors formulate an optimization problem to the estimation of the channel under a low-rank constraint without undersampling. However, full CSI is conveyed back to the Tx, which can be a bandwidth consuming process. The approach of [17] estimates the channel matrix by means of a matrix completion technique. The method is numerically investigated by considering different scattering environments for the MIMO channel model in an indoor scenario. Therein, the authors do not assume a lowrank channel model.
To the best of our knowledge, this paper is the first to propose the use of matrix completion in a general CSI feedback and reconstruction framework for massive MIMO systems. This work advances further than [9], [17] by presenting the problem in detail, while linking it to the massive MIMO paradigm. We also discuss two relevant application scenarios where the proposed approach is appealing. In the present work, upon reception of downlink pilots from Tx, for example a macro $\mathrm{BS}$, the receiver side (Rx), i.e., a micro-BS or a $\mathrm{UE}$, undersamples either the received pilot data matrix or the estimated channel matrix (depending on the chosen scheme), and feeds only a fraction of their entries back to the Tx. By capitalizing on matrix completion, the Tx recovers the downlink pilots for subsequent channel estimation, or directly reconstructs the downlink channel. Our results show that, when the channel matrix has a low-rank structure, the proposed feedback and reconstruction schemes yield accurate CSI estimation under a very small feedback overhead, which translates into a high energy efficiency and low complexity at the Rx, a desired feature for power-limited uplink transmissions. Thereby, the transmitter is able to design simple linear precoders [18], [19] and beamformers that consider a full-channel knowledge.

The contributions of this work can be summarized as follows:

- We propose a general framework to CSI feedback and reconstruction which capitalizes on matrix completion at the BS.

- We show the usefulness of the proposed framework in two application scenarios: wireless backhauling communications and multi-UEs uplink scenario. We discuss the performance in terms of bit error rate (BER) and goodput considering a minimum mean square error (MMSE) estimator.

- We formulate two feedback methods based on estimated channel undersampling (ECU) and direct data undersampling (DDU), which are respectively based on undersampling of the estimated channel and/or the received data matrix.

\section{A. Organization and Notations}

The rest of this paper is organized as follows. In Section II, we summarize the completion technique. Section III presents the system model. Section IV describes a general framework based on two operation modes. Two relevant application scenarios are presented in Section V. In Section VI, the simulation results are shown. Finally, Section VII brings some concluding remarks and perspectives.

a) Notation: Scalar, vector and matrix are denoted by lowercase letter, lowercase bold letter and uppercase bold letter, respectively. Superscripts ' $T$ ', and ' $H$ ' represent the transpose and the complex conjugate operation respectively. Nuclear norm of a matrix $\mathbf{Y}$ is denoted by $\|\mathbf{Y}\|_{*}$ defined as the sum of its singular values $\|\mathbf{Y}\|_{*} \triangleq \sum \sigma$. The Frobenius or Hilbert-Schmidt norm is denoted by $\|\mathbf{Y}\|_{F}$. The $|\langle\mathbf{A}, \mathbf{B}\rangle|$ is defined as $\operatorname{tr}\left(\mathbf{A}^{H} \mathbf{B}\right)$. 


\section{MAtrix Completion Problem}

In this section, we briefly explain the technique based on matrix completion. The full proofs of the exact and approximate recovery results to MC theory can be found in [20], [21] and an overview is found in [22].

The idea behind the MC problem is to recover an unknown low-rank matrix, exactly or approximately, from undersampled observations, with or without noise. Under some conditions, a desired (target) matrix can be recovered from the knowledge of only a fraction of its entries and by minimizing the nuclear norm (i.e., a sum of singular values) of the completed matrix [21].

More specifically, let $\mathbf{Y} \in \mathbb{C}^{n \times n}$ be the desired matrix to be reconstructed, where $n$ describes the matrix dimensions. We assume that only a few entries of $\mathbf{Y}$ are known. Moreover, let $\boldsymbol{\Omega}$ be a set whose elements are the known entries of $\mathbf{Y}$. For instance, if a tuple $(i j) \in \boldsymbol{\Omega}$, it means that the $(i j)$-th entry of $\mathbf{Y}$ is known.

To undersample $\mathbf{Y}$, let $P_{\boldsymbol{\Omega}}: \mathbb{C}^{n \times n} \rightarrow \mathbb{C}^{n \times n}$ denote the sampling operator defined by

$$
P_{\boldsymbol{\Omega}}(\mathbf{Y})= \begin{cases}y_{i j}, & \text { if }(i j) \in \mathbf{\Omega} \\ 0, & \text { otherwise, }\end{cases}
$$

where $y_{i j}$ is the $(i j)$-th entry of $\mathbf{Y}$. The sampling operator simply undersamples its input matrix, setting the output matrix entries corresponding to the unknown elements of $\mathbf{Y}$ to zero.

The reconstruction of the full matrix $\mathbf{Y}$ can be found by solving the optimization problem:

$$
\begin{aligned}
& \hat{\mathbf{Y}}=\arg \min _{\mathbf{X}} \operatorname{rank}(\mathbf{X}), \\
& \text { s.t. }\left\|P_{\boldsymbol{\Omega}}(\mathbf{X}-\mathbf{Y})\right\|_{F} \leq \gamma,
\end{aligned}
$$

where $\mathbf{X}$ is the matrix of variables and $\gamma \geq 0$ is a reconstruction error threshold. However, this is a nondeterministic polynomial-time (NP) hard-to-solve problem [23]. An alternative method is proposed to solve the problem using convex relaxation [24], as follows:

$$
\begin{aligned}
& \hat{\mathbf{Y}}=\arg \min _{X}\|\mathbf{X}\|_{*}, \\
& \text { s.t. }\left\|P_{\boldsymbol{\Omega}}(\mathbf{X}-\mathbf{Y})\right\|_{F} \leq \gamma .
\end{aligned}
$$

More specifically, despite the fact that nuclear norm minimization had long been observed to produce very low-rank solutions in practice [24], [25], theoretical results have shown that it produces the minimum rank solution.

Notice that the constraint in (4) makes sure that the entries of $\mathbf{X}$ corresponding to the known entries of $\mathbf{Y}$ are as close as possible. The optimization problem above is convex as both the objective and constraint are convex. Thus, it can be solved using general solvers available in the literature, e.g., [26]-[32].

It is worth mentioning that most $\mathrm{MC}$ algorithms make assumptions on the nature of matrix $\mathbf{Y}$. Herein, $\mathbf{Y}$ is assumed to have low-rank. When this assumption does not hold, a lowrank approximation is applied to $\mathbf{Y}$ in order to guarantee such an assumption. Additionally, the dimensions of the desired matrix must be large, e.g., in the order of hundreds. Otherwise, $\mathrm{MC}$ algorithms cannot provide a reasonable reconstruction [21].
Considering the MC theory in [20], [21], [25], [33], some simple hypotheses about the matrix $\mathbf{Y}$ are developed, which makes it recoverable. Let us describe these hypotheses briefly.

Consider the singular value decomposition (SVD) of $\mathbf{Y}$ as:

$$
\mathbf{Y}=\sum_{k=1}^{r} \sigma_{k} \mathbf{u}_{k} \mathbf{v}_{k}^{H},
$$

where $\sigma$ are the singular values, where $\sigma_{1} \geq \sigma_{2} \geq \ldots \geq \sigma_{r}$. $\left\{\mathbf{u}_{1}, \ldots, \mathbf{u}_{r}\right\} \in \mathbb{C}^{n}$ and $\left\{\mathbf{v}_{1}, \ldots, \mathbf{v}_{r}\right\} \in \mathbb{C}^{n}$ are two sets of orthogonal vectors. Consider $\mathbf{P}_{U}$ and $\mathbf{P}_{V}$ as the orthogonal projections onto the column and row space of $\mathbf{Y}$ respectively (singular vectors):

$$
\mathbf{P}_{U}=\sum_{i=1}^{r} \mathbf{u}_{i} \mathbf{u}_{i}^{H}, \quad \mathbf{P}_{V}=\sum_{i=1}^{r} \mathbf{v}_{i} \mathbf{v}_{i}^{H} .
$$

We define the matrix $\mathbf{E}$ as

$$
\begin{aligned}
& \mathbf{E} \triangleq \sum \mathbf{u}_{i} \mathbf{v}_{i}^{H}, \\
& \text { and } \mathbf{P}_{U} \mathbf{E}=\mathbf{E}=\mathbf{E P}_{V}, \quad \mathbf{E E}^{T}=\mathbf{P}_{V}, \quad \mathbf{E}^{T} \mathbf{E}=\mathbf{P}_{U} .
\end{aligned}
$$

To recover the matrix from part of its entries, the vectors $\mathbf{u}_{i}$ and $\mathbf{v}_{i}$ need to be "incoherent" in some sense. More specifically, the assumptions are as follows:

Assumption 1. There exists $\mu_{1}>0$ such that for all pairs $\left(a, a^{\prime}\right) \in n \times n$ and $\left(b, b^{\prime}\right) \in n \times n$,

$$
\begin{aligned}
& \left|\left\langle\mathbf{e}_{a}, \mathbf{P}_{U} \mathbf{e}_{a^{\prime}}\right\rangle-\frac{r}{n_{1}} 1_{a=a^{\prime}}\right| \leq \mu_{1} \frac{\sqrt{r}}{n} \\
& \left|\left\langle\mathbf{e}_{b}, \mathbf{P}_{V} \mathbf{e}_{b^{\prime}}\right\rangle-\frac{r}{n_{2}} 1_{b=b^{\prime}}\right| \leq \mu_{1} \frac{\sqrt{r}}{n},
\end{aligned}
$$

where $\mathbf{e}$ is a canonical vector and $1_{E}$ the indicator function of an event $E$, e.g. $1_{a=a^{\prime}}$ is equal 1 to $a=a^{\prime}$ and 0 to $a \neq a^{\prime}$.

Assumption 2. There exists $\mu_{2}>0$ such that for all $(a, b) \in$ $n \times n$

$$
\left|E_{a b}\right| \leq \mu_{2} \frac{\sqrt{r}}{n} .
$$

If the above assumption holds, we say that the matrix $\mathbf{Y}$ obeys the strong incoherence property with parameter $\mu=$ $\max \left(\mu_{1}, \mu_{2}\right)$.

Based on these hypotheses, [33] proposes the following theorem:

Theorem 1: Let $\mathbf{Y} \in \mathbb{C}^{n \times n}$ be a fixed matrix of rank- $r$ obeying the strong incoherence property with parameter $\mu$. Suppose we observe $m$ entries of $\mathbf{Y}$ with locations sampled uniformly at random. Then, there is a positive numerical constant $\mathrm{C}$ such that if

$$
m \geq C \mu^{2} n r \log _{10}(n)^{\varrho},
$$

where $\varrho$ is a positive number, then $\mathbf{Y}$ is the unique solution to (4) with probability at least $1-n^{-3}$, i.e., with high probability, the nuclear-norm minimization recovers all the entries of $\mathbf{Y}$ without error.

More details on the incoherence property discussed in Assumptions 1 and 2, and its relation to the completion of the channel matrix are given in the Appendix. 


\section{SySTEM MODEL}

Consider a wireless communication system, where a BS represents the Tx and the Rx can be represented by others BSs or the UEs. The Tx and Rx are equipped with $M_{T}$ and $M_{R}$ antennas, respectively. During the downlink training phase, the BS sends pilot sequences of length $N_{T}$ to the Rx. The received signal at the Rx can be expressed as:

$$
\mathbf{Y}=\mathbf{H S}^{T}+\mathbf{N} \in \mathbb{C}^{M_{R} \times N_{T}},
$$

where $\mathbf{H} \in \mathbb{C}^{M_{R} \times M_{T}}$ is the channel matrix, $\mathbf{S} \in N_{T} \times M_{T}$ contains the pilot sequences, and $\mathbf{N} \in \mathbb{C}^{M_{R} \times N_{T}}$ the additive white Gaussian noise term.

The channel model is expressed as a sum of a finite (small) number of specular paths, as follows:

$$
\mathbf{H}=\frac{1}{\sqrt{P}} \sum_{p=1}^{P} \alpha_{p} \mathbf{a}_{r}\left(\theta_{p}\right) \mathbf{a}_{t}\left(\delta_{p}\right)^{T},
$$

where $P$ defines the number of paths, $\alpha_{p}$ is the fading amplitude associated with the $p$-th path, and the steering vector is defined as [34]:

$$
\begin{aligned}
& \mathbf{a}_{r}\left(\theta_{p}\right) \triangleq\left[1, e^{-j 2 \pi \frac{d}{\lambda} \cos \left(\theta_{p}\right)}, \ldots, e^{-j 2 \pi \frac{\left(M_{R}-1\right) d}{\lambda} \cos \left(\theta_{p}\right)}\right]^{T}, \\
& \mathbf{a}_{t}\left(\delta_{p}\right) \triangleq\left[1, e^{-j 2 \pi \frac{d}{\lambda} \cos \left(\delta_{p}\right)}, \ldots, e^{-j 2 \pi \frac{\left(M_{T}-1\right) d}{\lambda} \cos \left(\delta_{p}\right)}\right]^{T},
\end{aligned}
$$

where $d$ is the inter-element antenna spacing, $\lambda$ is the wavelength, $\theta_{p}, \delta_{p} \in[0, \pi]$ are the angle of arrival (AoA) and angle of departure (AoD), respectively, associated with the $p$-th path. Furthermore, the channel model described in (13) is a stochastic multipath channel [35].

Many discussions about the channel have been raised over the last years. This paper relies on the assumption of a finite scattering environment, i.e., the number of multipaths is smaller compared to the number of antennas. Finite scattering models are usually adopted for millimeter-wave (mmWave) scenarios [36]. For example, in $60 \mathrm{GHz}$ with massive MIMO, the high path loss will lead the propagation to be only the line-of-sight (LOS) or the first and second order reflections, such that the number of incoming signal paths is limited [37], [38]. Other scenarios also present a small number of scatterers, e.g., when a BS is equipped with a large number of antennas located in an elevated position with few scatterers around (e.g., on the top of a high building, a dedicated tower, or a unmanned aerial vehicle platform), and the channel is mainly characterized by rich local scatterers around the UE (e.g., the classical one-ring model [39], [40]). Moreover, the use of antennas that are highly directive further reduces the number of surrounding scatterers. Hence, the angular spread seen by the BS is small, and the number of incoming signal paths is limited. Also, several related works assume similar insights [7], [35], [41]-[47]. Thereby, for the considered scenarios in this work, the channel matrix is assumed to have low-rank.

On the other hand, a generalization of the standard lowrank matrix completion problem in which the matrix rank can be quite high or full-rank was made in [48]. It is true only under some mild conditions, which are beyond the scope of this work.

\section{FRAMEWORK AND OPERATION MODES}

\section{A. General Problem}

In this section, we are interested in solving a general feedback problem. More specifically, to fully utilize the array gains of massive MIMO, the CSI must be available at the transmitter side. Let us focus on communication systems based on FDD that require some form of channel feedback. This type of system faces a feedback overhead problem when assuming a massive number of antennas, since CSI feedback overhead scales linearly with the number of antennas. Hence, the success of FDD-based massive MIMO systems implies low complexity feedback schemes that are bandwidth-efficient.

To illustrate this problem, consider a conventional feedback scheme in a MIMO system, where the full channel matrix is conveyed from the Rx back to the Tx. The number of bits to be feed back is given by $\nu=\gamma \log _{2}\left(M_{R} M_{T}\right)$, where $\gamma$ depends on the accuracy of channel information at the Rx. Assuming $M_{T}=M_{R}=100$ and $\gamma=3$, the Rx needs $\nu=39.8631$ bits to convey the full CSI to the Tx.

In this context, we resort to MC techniques to solve the feedback overhead problem, by exploiting the low-rank nature of the MIMO channel. The framework of the underlying idea is shown in Fig. 1.

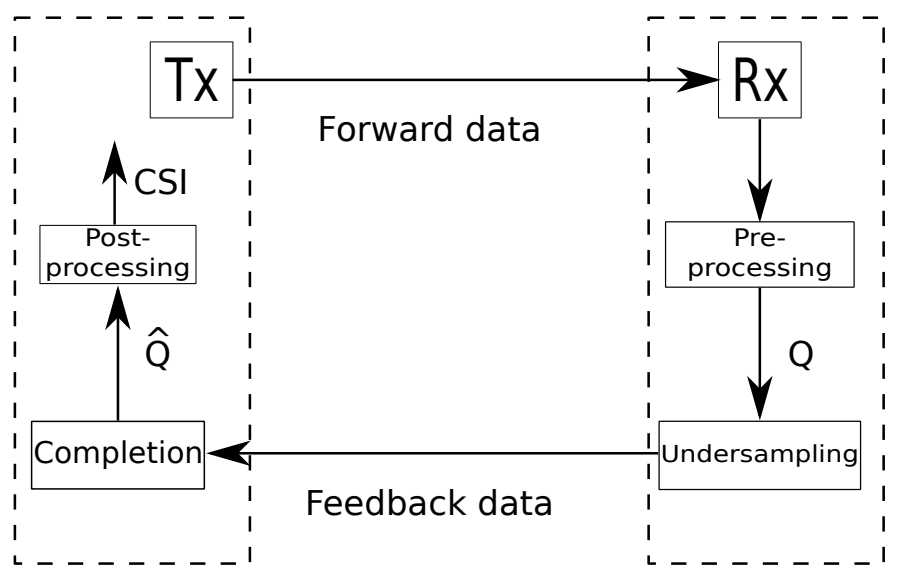

Fig. 1. Framework structure.

The process is initialized with the Tx sending a forward data to $\mathrm{Rx}$ and, after a pre-processing, Rx sends a reduced feedback data to Tx assuming an undersampling factor $m$. The parameter $m$ defines the fraction of entries in the matrix $\mathrm{Q}$ that is sent back to the Tx via a feedback channel. For example, when considering $m=0.1$, we are sending $10 \%$ of total entries of $\mathbf{Q}$. Furthermore, if the technique to obtain the CSI is based on pilot symbols, the forward data is a training sequence and the pre-processing block is the channel estimator.

After receiving forward data sent by $\mathrm{Tx}, \mathrm{Rx}$ may first pre-process such forward data. The result is the matrix $\mathbf{Q}$. Then, Rx undersamples $\mathbf{Q}$ and the undersampled data are sent through the feedback channel ${ }^{1}$. Tx receives the data and then

\footnotetext{
${ }^{1}$ We assume that the uplink channel is error-free.
} 
applies MC to this matrix. At the end of this step, the matrix $\mathbf{Q}$ is in turn reconstructed as $\hat{\mathbf{Q}}$. Finally, after a post-processing (channel estimation), the CSI is made available at Tx.

\section{B. Operation Modes}

Herein, two types of feedback data are visualized: (a) the received signal matrix $\mathbf{Y}$, or (b) the estimated channel matrix $\hat{\mathbf{H}}$. Based on this, we define two operation modes:

- DDU: in this mode, a fraction of the received signal $\mathbf{Y}$ is the feedback data, which means that $\mathbf{Q}=\mathbf{Y}$. Therefore, there is no pre-processing step. Consequently, $\mathrm{Rx}$ operates as a simple sampling device. Tx applies the completion algorithm to reconstruct $\mathbf{Y}$. Due to the noise, the matrix $\mathbf{Y}$ is actually full-rank. Eventually, Tx obtains a filtered version of $\mathbf{Y}$, from which the channel matrix $\hat{\mathbf{H}}$ is estimated as a post-processing step.

- ECU: in this mode, a fraction of the estimated channel $\hat{\mathbf{H}}$ is the feedback data, which means that $\mathbf{Q}=\hat{\mathbf{H}}$. Therefore, the pre-processing is a channel estimation step performed by the Rx taking into account the received signal $\mathbf{Y}$. At Tx side, there is no post-processing step.

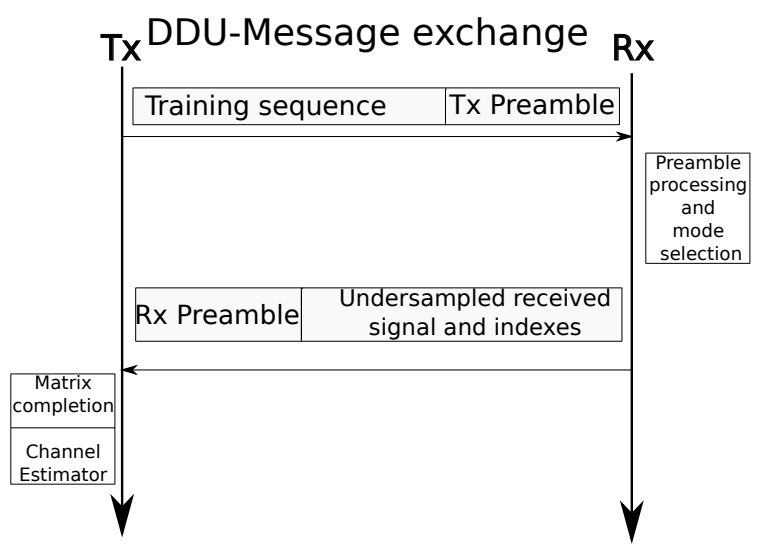

Fig. 2. Message exchange and processing between Tx and Rx. In the DDU mode, a fraction of the received signal is sent by the receiver via a limited feedback channel.

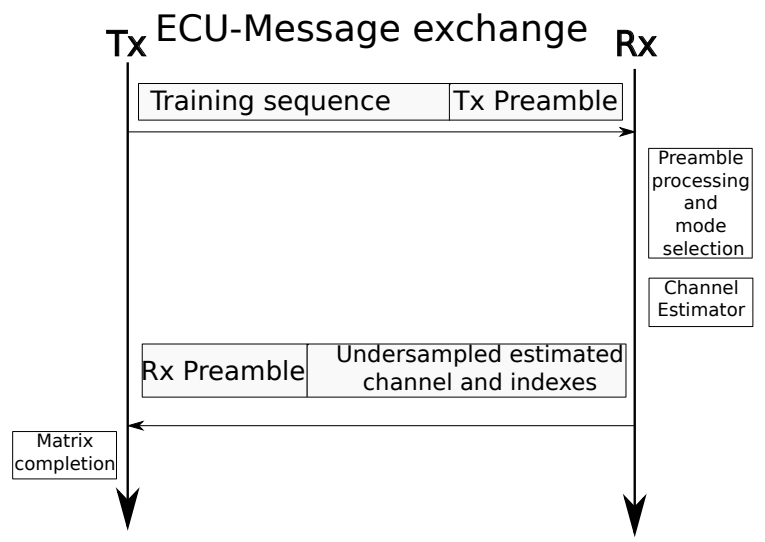

Fig. 3. Message exchange and processing between Tx and Rx. In the ECU mode, a fraction of the estimated channel is sent by the receiver via a limited feedback channel.

The main steps of the systematic message exchange between Tx and Rx are illustrated in Fig. 2 and Fig. 3 for the DDU and ECU operating modes, respectively, and are summarized below:

- Tx sends the training sequence to Rx with a Tx preamble;

- Rx reads the Tx preamble. Then, Rx selects the mode and the undersampling factor. The extracted samples and index set are fed back to Tx along the with Rx preamble;

- Tx reads the Rx preamble. Then, Tx recovers the full data by using a matrix completion algorithm.

Basically, the preambles contain information about the predefined mode selection and undersampling factor, as described in Table I. In Tx preamble a sequence of bits $\mathrm{A}=[\mathrm{A} 1, \mathrm{~A} 2, \mathrm{~A} 3]$ is used to choose between the DDU and ECU modes. A third mode, namely, full mode, is also covered, which coincides with the standard feedback scheme. The Rx preamble contains a sequence of bits $\mathrm{B}=[\mathrm{B} 1, \mathrm{~B} 2, \mathrm{~B} 3]$ to inform $\mathrm{Tx}$ what mode is in use. The bits $\mathrm{A} 1$ and $\mathrm{B} 1$ are reserved for extra modes and possible future implementations.

Note that the main difference between DDU and ECU modes is associated with the type of feedback data in Fig. 4. Parameter $\mathrm{T}$ means the forward data time, $\mathrm{T}_{\mathrm{f}}$ means the feedback data time, $\mathrm{T}_{\mathrm{us}}$ is the time needed to begin the undersampling, $\tau$ is the total transmission interval, $\mathrm{T}_{\mathrm{DDU}}$ and $\mathrm{T}_{\mathrm{ECU}}$ stand for the data transmission time in DDU and ECU modes, respectively. In the DDU mode, the feedback data is the training sequence. Note that in the DDU mode the Rx does not need to wait the reception of the entire incoming signal. On the other hand, in the ECU mode, the feedback data is the estimated channel. Thus, the Rx needs to wait the reception of the entire incoming signal to estimate the channel. Therefore, $\mathrm{T}_{\mathrm{DDU}} \geq \mathrm{T}_{\mathrm{ECU}}$.

TABLE I

DESCRIPTION OF THE TX PREAMBLE.

\begin{tabular}{|c|c|c|c|}
\hline \multicolumn{3}{|c|}{ Bits } & Downlink \\
\hline \multirow[t]{4}{*}{ A1 } & A2 & A3 & Tx preamble \\
\hline & 0 & 0 & $\begin{array}{l}\text { Full Mode - Defines that either DDU, ECU or full- } \\
\text { CSI feedback (baseline) can be chosen, Rx selects } \\
\text { the mode of operation. }\end{array}$ \\
\hline & 0 & 1 & DDU mode - Direct data undersampling; \\
\hline & 1 & 0 & ECU mode - Estimated channel undersampling. \\
\hline \multicolumn{3}{|c|}{ Bits } & Uplink \\
\hline \multirow[t]{4}{*}{ B1 } & $\mathrm{B} 2$ & B3 & Rx preamble \\
\hline & 0 & 0 & Full Mode \\
\hline & 0 & 1 & $\overline{\text { DDU mode }}$ \\
\hline & 1 & 0 & ECU mode \\
\hline
\end{tabular}

The DDU mode has low computational complexity, low latency, and low energy consumption $(\mathrm{Rx})$ compared to the ECU mode, since it does not estimate the channel. If a fullduplex capability is available, which is more likely to be beneficial in the wireless backhaul scenario [49], Rx can feedback data to Tx while it is still receiving the signal $\mathbf{Y}$. Thus, Tx can obtain CSI more quickly and possibly use the time resources more efficiently by transmitting more data in the same data transmission interval, as illustrated in Fig. 4. Besides, the computational burden associated with channel estimation is moved to the Tx side, which means that Rx saves energy as it does not need to estimate the channel $\mathbf{H}$. On the other hand, according to (12), $\mathbf{Y}$ contains an additive white 

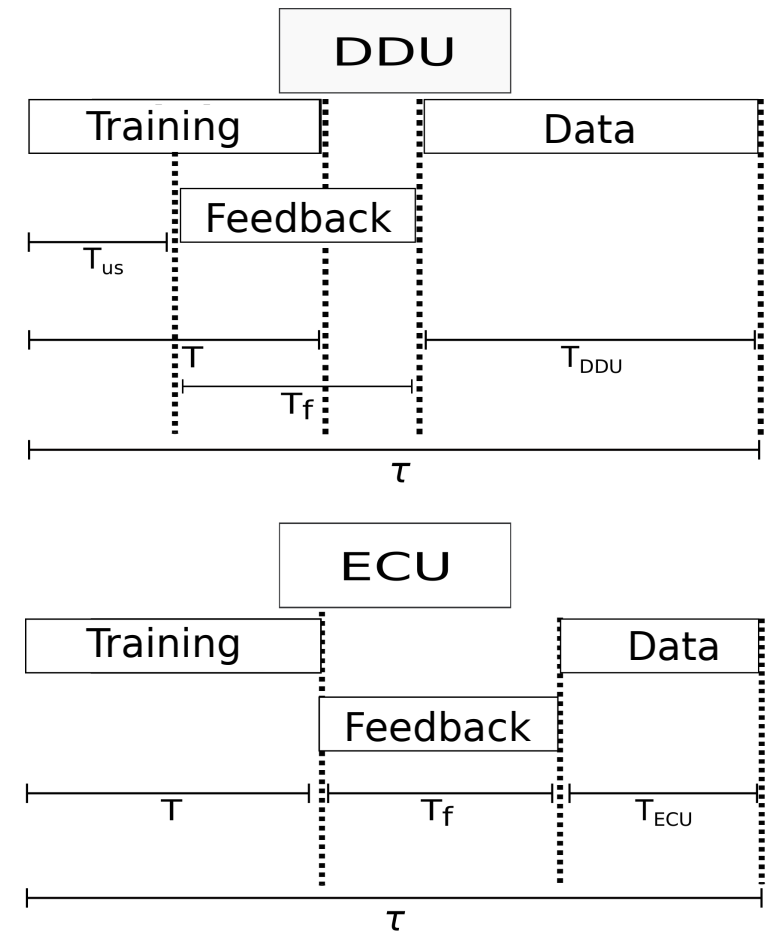

Fig. 4. Difference between DDU and ECU in the time domain.

noise term, which turns it a full-rank matrix. Even after a lowrank approximation within the matrix completion procedure, the reconstructed matrix $\hat{\mathbf{Y}}$ is still corrupted by the additive noise in the DDU, which is adverse for channel estimation. In other words, while the ECU estimates the channel directly from $\mathbf{Y}$ and reconstructs $\hat{\mathbf{H}}$, which is already a low-rank matrix, the DDU makes use of low-rank approximations of $\mathbf{Y}$ to estimate the channel from a corrupted version of $\mathbf{Y}$.

The advantages and disadvantages of the two data feedback modes lead us to think of a tradeoff between DDU and ECU. If some estimation error is acceptable, DDU can be preferable as it provides energy saving on the Rx side. Conversely, if CSI must be accurate on the Tx side, ECU might be preferable ${ }^{2}$. Interestingly, in a scenario with very high signal-to-noise ratio (SNR), DDU is no longer affected by noise and it would perform as good as ECU. On the other hand, if a full-duplex capability is not available, DDU mode can no longer efficiently use the time resources.

To better exemplify the proposed framework structure, in what follows we illustrate the framework with two application examples: wireless backhauling and a clustered multi-UE scenario.

\section{Application Scenarios}

In this section, we apply the proposed framework in two relevant scenarios. The first is a new problem imposed in massive MIMO systems with heterogeneous networks supporting a macro-cell layer with additional small cells, where wireless backhauling communications take place between the macroBS and a micro-BS. The second one is related to multi-user

\footnotetext{
${ }^{2}$ The simulation results in Section VI confirm this claim.
}

channel estimation in a clustered multi-UE massive MIMO system.

\section{A. Application Scenario 1: Wireless backhauling}

In dense cell deployments, wired backhaul becomes expensive or even infeasible due to the large number of network nodes to be connected. As an alternative to overcome this limitation, millimeter-wave wireless backhaul, e.g., in $60 \mathrm{GHz}$, can be adopted [50]-[52].

Here we assume a wireless backhaul system in which Tx and $\mathrm{Rx}$ are represented by a macro-BS and micro-BS, respectively. The Tx and Rx are equipped with a massive MIMO array and operate in FDD. Figure 5 illustrates an application of the proposed framework to the wireless backhauling scenario. In addition, we assume a small number of dominant scatterers and local scattering is limited. By the superposition of a few reflected signals, the channel matrix has low-rank and follows the model expressed in (13). This is a typical assumption for channels in millimeter-wave bands [53], i.e., there is a LOS path and only a few number of multipaths.

\section{B. Application Scenario 2: Multi-UEs}

Consider a multi-UEs scenario where the UEs are spread across $C_{l}$ spatial regions or clusters. We define a cluster as the association of the channel matrix with a spatial subspace. That is, UEs inside a cluster have approximately the same spatial channel. It worth mentioning that such clustered multi-UEs scenario can be exploited by scheduling and linear precoding schemes [41], [54], [55].

More specifically, let $\mathrm{Tx}$ be a massive BS equipped with $M_{T}$ antennas, and let Rx denote $K$ UEs equipped with $M_{R}$ antennas each. Figure 6 shows the systemic view on how the proposed feedback and reconstruction framework can be applied in the multi-UEs scenario. We assume a poor scattering scenario with $P$ specular multipaths which $P \ll M_{T}$. Also, we assume that a cluster of UEs is formed when those UEs share the same set/cluster of scatterers. The UEs are spatially close to one another in a dense hot-spot area, and, thus, are grouped in a cluster.

Different from the wireless backhauling scenario, in the multi-UEs scenario the feedback data are spread over UE clusters. Thus, in order to apply MC, the BS folds all the received feedback data as a global matrix ${ }^{3}$. For building the global channel matrix, we follow the channel model described in (13) so that

$$
\mathbf{H}_{T}=\left[\mathbf{H}_{1}^{T}, \mathbf{H}_{2}^{T}, \ldots, \mathbf{H}_{C_{l} K}^{T}\right]^{T} \in \mathcal{C}^{C_{l} K M_{R} \times M_{T}} .
$$

The global received signal matrix is then given by

$$
\mathbf{Y}=\left[\mathbf{Y}_{1}^{T}, \mathbf{Y}_{2}^{T}, \ldots, \mathbf{Y}_{C_{l} K}^{T}\right]^{T} \in \mathcal{C}^{C_{l} K M_{R} \times N_{T}}
$$

which concatenates the contributions from all UEs.

We consider that each cluster of UEs is far from the BS as showed in Fig. 6, while the scatterer is near to UEs. Thereby,

\footnotetext{
${ }^{3}$ We consider that the BS can separate each UE perfectly (i.e. no intra-cell interference).
} 


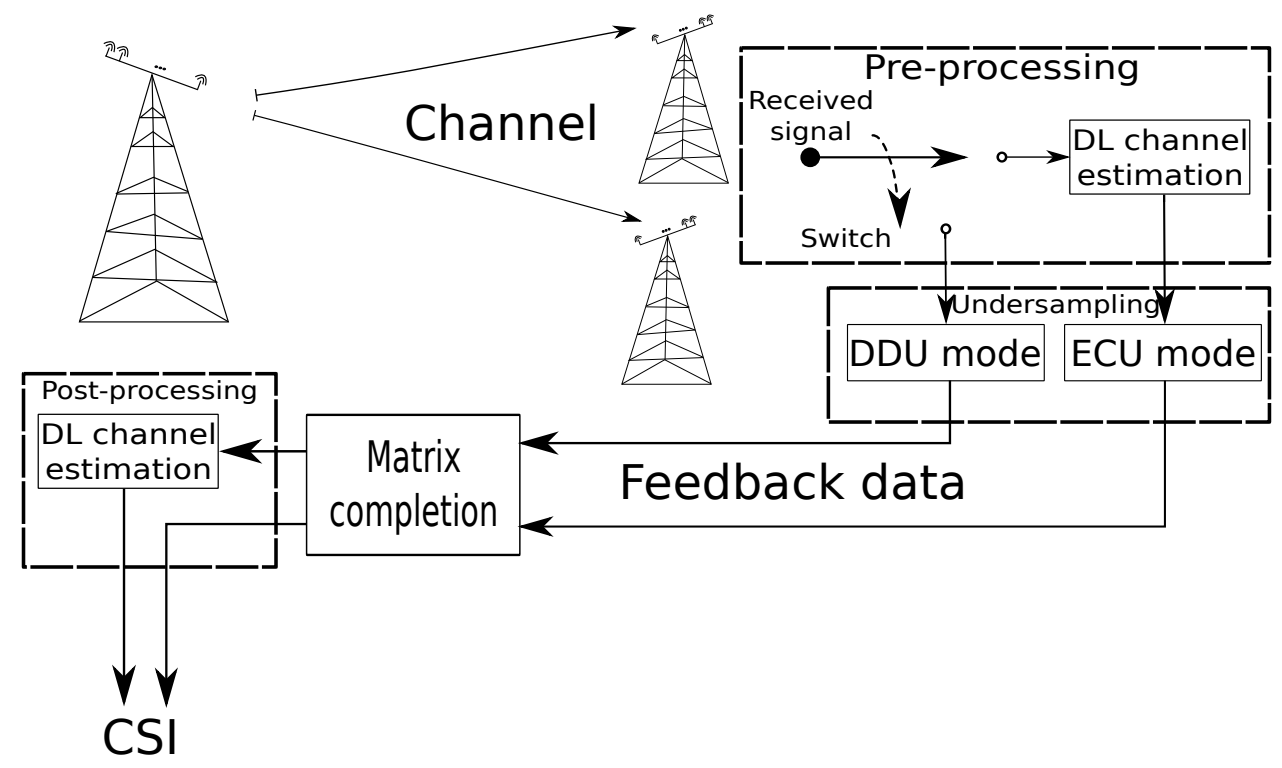

Fig. 5. This figure presents an scenario of application in the wireless backhauling scenario. Two types of feedback data for the framework are presented. Tx is the macro-BS and Rx can be one or more micro-BSs equipped with a massive array.

the following model is adopted to describe the $k$-th UE channel matrix:

$$
\mathbf{H}_{k}=\frac{1}{\sqrt{P}} \sum_{p=1}^{P} \alpha_{p} \mathbf{a}_{r}\left(\theta_{p}+\gamma_{k}\right) \mathbf{a}_{t}\left(\delta_{p}\right)^{T},
$$

where $\gamma_{k}$ represents the $k$-th UE angular deviation from the mean angle $\theta_{p}$ and is modeled as a zero-mean Gaussian random variable with variance $\sigma_{a}^{2}$ for each UE. The UEs resultant channels in each cluster are correlated, implying a low-rank global channel matrix [7], [41], [56].

\section{Simulations Results}

In this section, we divide our simulations according to the two application examples discussed in the previous section. The reconstruction error of the full data matrix (DDU) is evaluated at a given Monte Carlo run, by computing the normalized mean square error (NMSE) defined as $\|\mathbf{Y}-\hat{\mathbf{Y}}\|_{F}^{2} /\|\mathbf{Y}\|_{F}^{2}$, where $\hat{\mathbf{Y}}$ is an estimate of the full data matrix. The NMSE measure for the ECU is similarly defined. The NMSE results represent an average over 100 runs, and are plotted as a function of the factor $m$.

In this simulation, we assume the Tx is equipped with a uniform linear array (ULA). A very accurate reconstruction is assumed when the NMSE is around $10^{-4}$ [57]. Furthermore, the ECU scheme considers a MMSE channel estimator [58]. The transmitted symbols are mapped into the binary phase shift keying (BPSK) constellation and $N_{T}=M_{T}$. The estimated channel NMSE is calculated as: $\|\mathbf{H}-\hat{\mathbf{H}}\|_{F}^{2} /\|\mathbf{H}\|_{F}^{2}$, in which $\hat{\mathbf{H}}$ is given by the MMSE estimator.

In the reconstruction problem described in Fig. 1, we use the two algorithms proposed in [32]. The first is based on non-convex algorithm (NCon) while the second is the fast numerical soft threshold algorithm (FST). We tested other algorithms based on singular value thresholding (SVT) [27], subspace evolution transfer (SET) [28], low-rank matrix fitting (LMaFit)[59], iteratively reweighted least squares recovery for matrix completion (IRLS-M) [31], and another one based on grassman rank-one update subspace estimation (Grouse) [30], [57]. However, since the focus of our proposal is on the channel feedback and reconstruction framework, we selected the ones in [32] which have demonstrated to be the preferable solutions under the NMSE criterion.

\section{A. Wireless backhauling scenario}

Consider a system with $M_{T}=M_{R}$, where the $\mathrm{Rx}$ is equipped with a ULA. Figure 7 describes a street scenario (e.g., wireless backhauling between a macro-BS and a micro$\mathrm{BS})$. We consider the street with 10 meters width and 100 meters length. We assume $P=5$, which means that the channel matrix rank equals 5. The fading amplitude $\alpha_{p}$ in (13) is calculated using the path loss (PL) model adopted in [53] (c.f. Table 1). More specifically, it can be calculated as $\mathrm{PL}=16 \pi^{2}\left(\frac{R}{\lambda}\right)^{n}$ for a given range distance $R$, wavelength $\lambda$, and PL exponent $n$. We assume a Tx-Rx distance equal to $R=100$ meters, a carrier frequency of $60 \mathrm{GHz}$, and PL exponent $n=2.5$, representing a LOS scenario. The channel is composed by the sum of a LOS path and four non-LOS paths with one and two reflections according to Fig. 7.

In Fig. 8 the NMSE is plotted by varying the number of antennas. We compare the ECU and DDU modes with SNR equals $30 \mathrm{~dB}$ in order to measure the reconstruction error using the NCon algorithm and MMSE estimator. At $m=0.1$, even under high number of antennas, the minimal error is around $10^{-3}$. At $m=0.2$, the NMSE has linear decrease with the number of antennas. Note that, in this scenario, using $64 \mathrm{Tx} / \mathrm{Rx}$ antennas is enough to find a very accurate channel reconstruction (i.e., NMSE $=10^{-4}$ ). When $m=0.3$, the NMSE decreases fastly, and around 48 antennas is enough for achieving a very good channel reconstruction. 


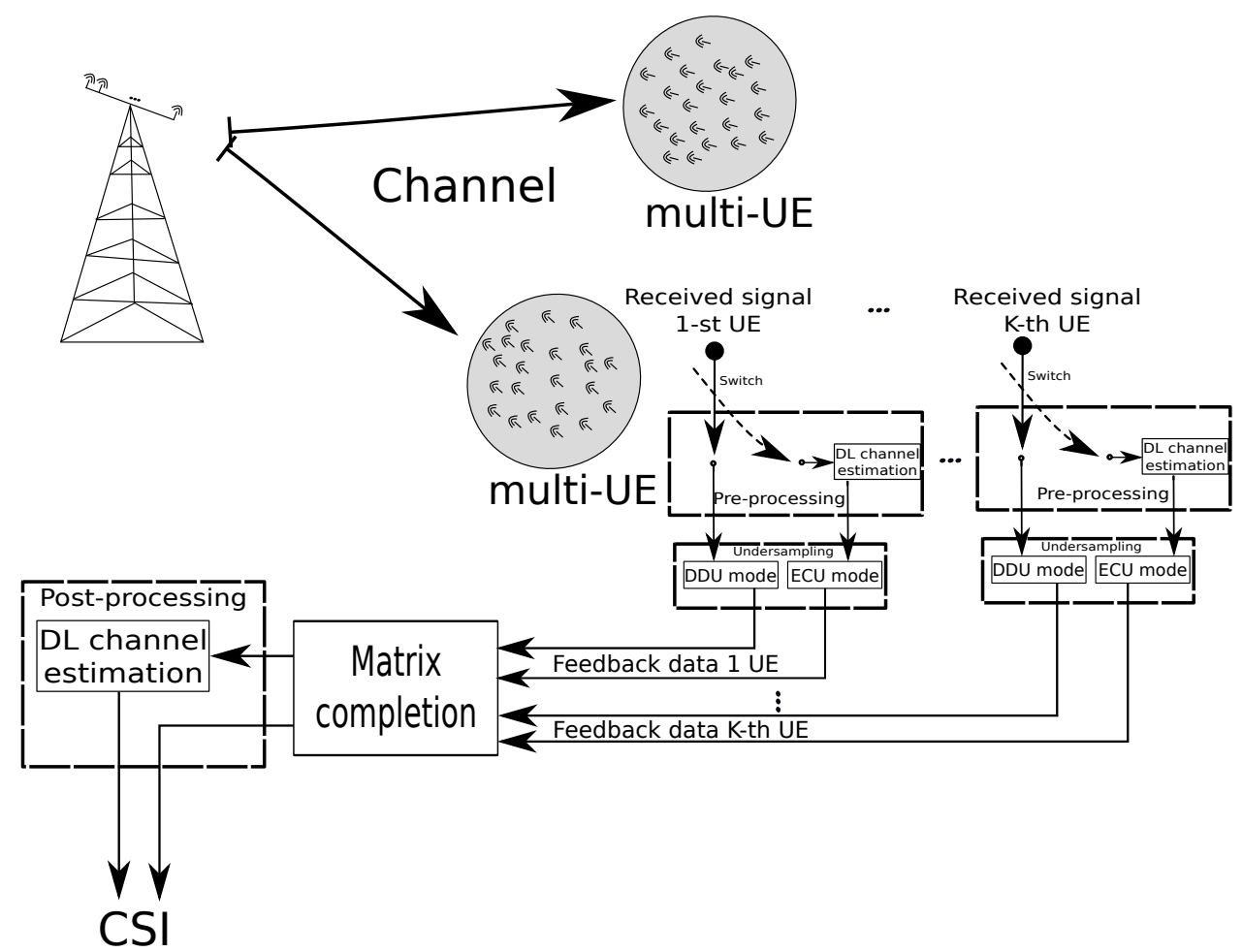

Fig. 6. This figure presents an application scenario in the multi-UEs scenario where each UE has a single (but not limited to) antenna. UEs are assumed to be close to one another (clustered UEs).

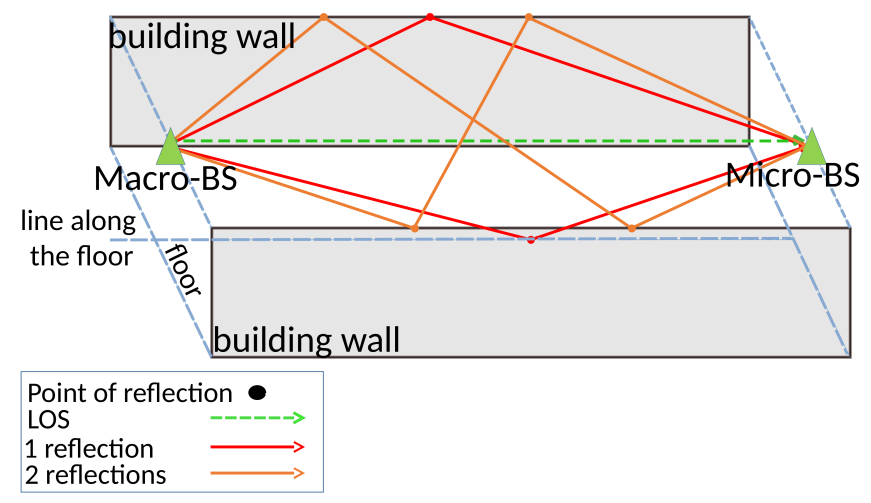

Fig. 7. Wireless backhaul scenario.

In Fig. 9, we present the NMSE results by applying the matrix completion framework. The MMSE curve is the estimation error before the undersampling procedure. This result provides a lower bound, since this curve represents the case of a conventional feedback (without reconstruction errors). We assume that the CSI is recovered with an undersampling factor $m \in\{0.05,0.15,0.35,0.55,0.65\}$ for DDU and ECU. Note that, the performance of ECU is equal to, or better than DDU in most simulations, since $Y$ contains an additive white noise term for DDU. The ECU achieves the an almost-perfect reconstruction with an SNR equals $35 \mathrm{~dB}$ with $m=0.15$, i.e., only $15 \%$ of samples are enough to recover the channel, thus saving $85 \%$ compared to the conventional full channel feedback. For $m \in\{0.35,0.55,0.65\}$, the ECU achieves its maximal performance, which is similar

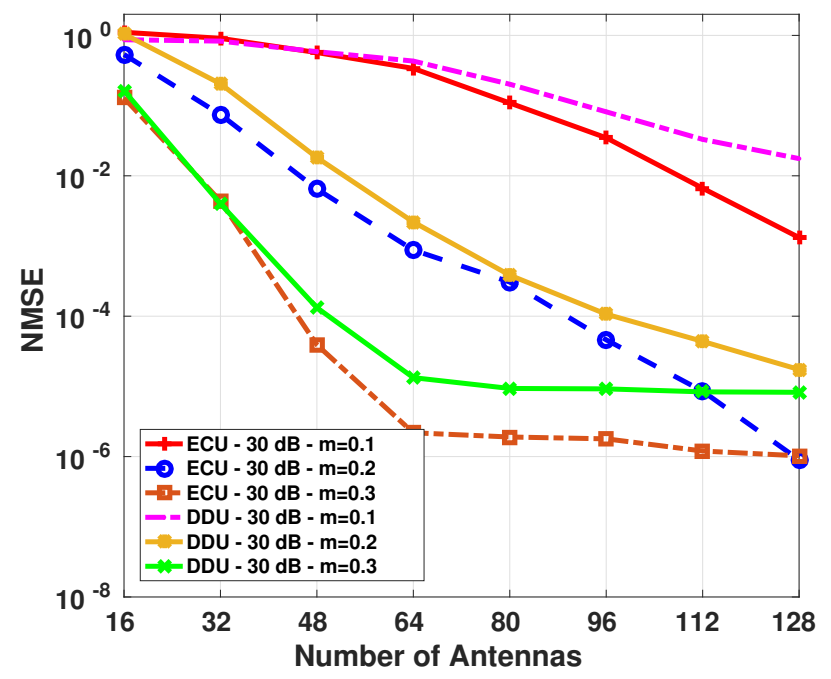

Fig. 8. NMSE performance for different antennas number with $m \in$ $\{0.1,0.2,0.3\}$ for ECU and DDU.

to that of conventional full channel feedback (MMSE). The DDU obtains an almost-perfect reconstruction with an SNR equals $28 \mathrm{~dB}$ with $m=0.35$. The DDU has performance losses around $3 \mathrm{~dB}$ compared to conventional full channel feedback, in the range of SNR between 10 and $30 \mathrm{~dB}$ for $m \in\{0.55,0.65\}$. Note that, in Fig. 9, the performance of proposal is limited by the MMSE estimator error. 


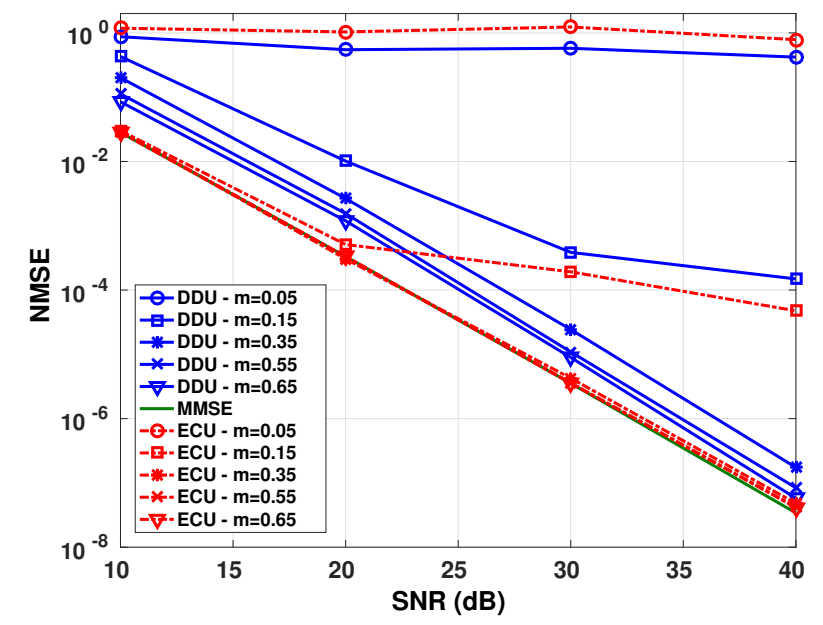

Fig. 9. NMSE results for DDU and ECU.

\section{B. Multi-UEs scenario}

In this section, we evaluate the performance of the proposed schemes, considering the Tx as BS equipped with $M_{T}=100$ and the $\mathrm{Rx}$ as $K$ multi-UEs with the same number of $M_{R}$ antennas. For instance, assuming $C_{l}=2$, Fig. 6 means that we are considering two independent clusters of multi-UEs. We assume $P=1$, and the angular deviation $\gamma_{k}$ of each UE has a variance $\sigma_{a}^{2}=5$.

In the first, second and third simulation experiments, we consider the channel is perfectly estimated. Figure 10 considers an example that arises in millimeter-wave communications, where both BS and UE have a large number of antennas. In this case, we generate the correlated channel with $K=C_{l}=1$, i.e., the channel has rank-one. Figure 10 shows the performance of the ECU to $M_{R}=M_{T}=100$. Note that the ECU converges with less than $m=0.075$ to perfect recovery, saving more than $92.5 \%$ of the feedback information. In this case, the NCon has a better performance for $m=0.1$.

In Fig. 11, we consider a scenario with $K=5, M_{R}=$ 10 and $C_{l}=2($ rank=2). The performance results show that the ECU can recover the channel matrix with very accurate reconstruction in $m=0.125$, i.e., less of $15 \%$ using NCon. Note that compared to Fig. 10, the performance is worse than that with $m=0.1$ for both algorithms because rank equals 2 . However, the NCon achieves an almost perfect reconstruction. The FST can achieve an almost perfect reconstruction with $m=0.175$. Henceforth, we use NCon to solve the ECU in all simulations.

In Fig. 12, we compare the performances of ECU and DDU. In this case, we limit the results to FST, because it showed a better performance compared to NCon for DDU. Observe that DDU has a reasonable recovery $\left(\mathrm{NMSE}=10^{-3}\right.$ ) requiring at least $\mathrm{SNR} \geq 20 \mathrm{~dB}$ and $m=0.3$. Specifically, it achieves a very accurate reconstruction with $\mathrm{SNR}=40 \mathrm{~dB}$ and $m=0.35$. In fact, as shown in Fig. 12 and in Fig. 9, the MC performance is limited by noise [33], [57], explaining the worst performance of DDU compared to ECU.

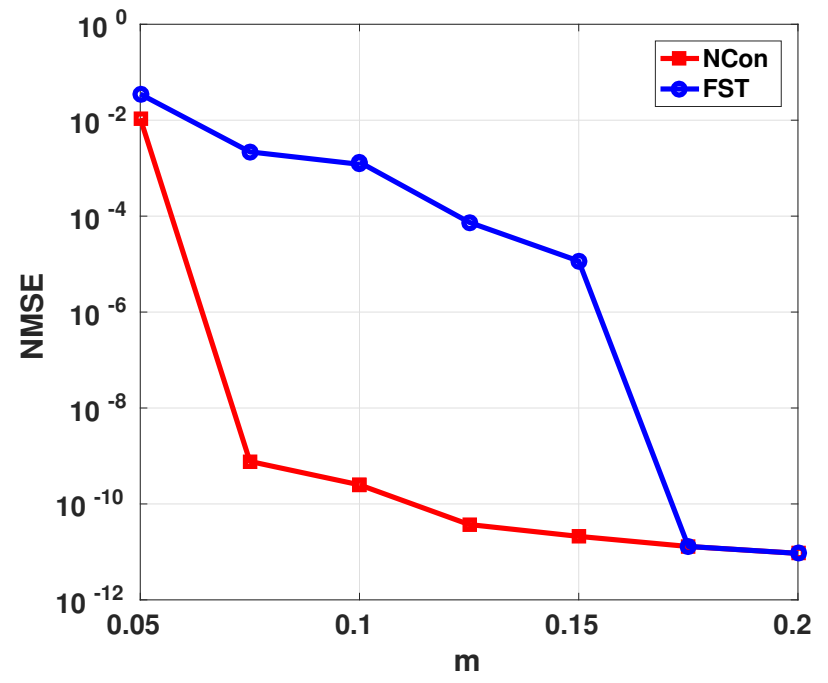

Fig. 10. Performance for the channel recovery with the ECU for UE with $M_{R}=100$.

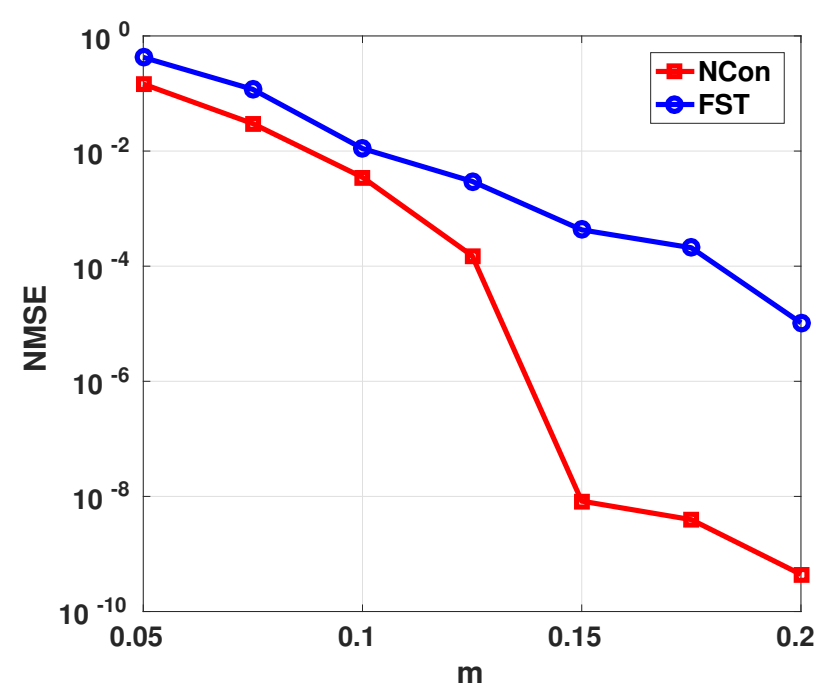

Fig. 11. Performance for the channel recovery with the ECU, $K=5, M_{R}=$ 10 , and $C_{l}=2($ rank=2).

In order to evaluate the performance and robustness of the proposed framework under a practical scenario, we consider a controlled error in the CSI and the MRT precoder [18]. The imperfect CSI is modeled as $\hat{\mathbf{H}}=\mathbf{H}+\mathbf{E}$, where $\mathbf{E} \approx \mathcal{N}\left(0, \sigma_{e}\right)$ and $\sigma_{e} \in\{0.1,0.05,0.01\}$. Firstly, let us show the results for the imperfect CSI in Fig. 13. Under a lower level of estimated channel error $\left(\sigma_{e}=0.01\right)$, the performance is the same of the perfect channel. Under $\sigma_{e} \in\{0.05,0.1\}$, the performance losses when BER equals $10^{-2}$ is $2 \mathrm{~dB}$ and $5 \mathrm{~dB}$, respectively.

In Fig. 14, we show the BER performance of MRT applying the ECU with $K=10, M_{R}=1$ and $C_{l}=10$ (rank=10). We provide simulation results to $m \in\{0.1,0.2,0.3,1\}$. The performance of the algorithm to very accurate reconstruction is achieved with $m=0.3$ which is when the performance of the proposal with MRT is the same as that of an accurate CSI. When $m \in\{0.1,0.2\}$ the performance loss compared to the 


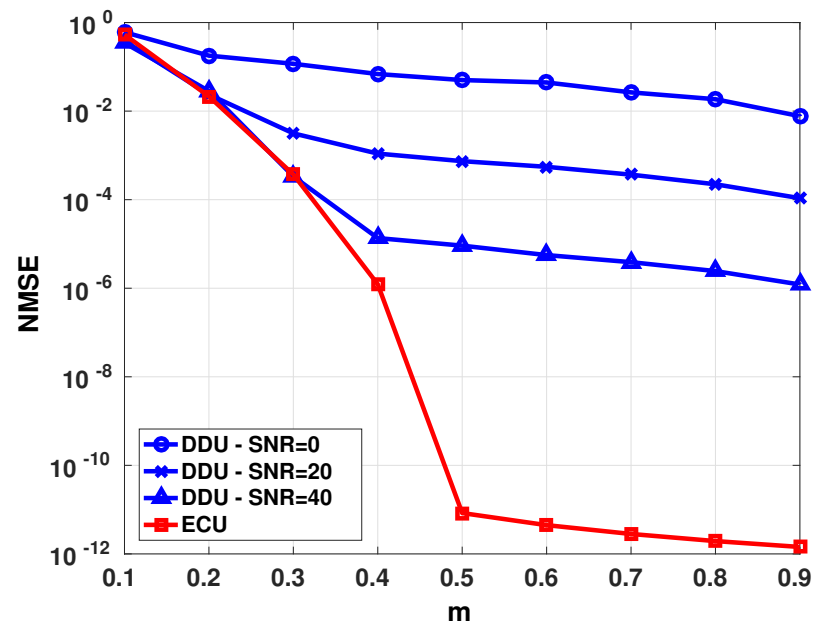

Fig. 12. Performance for the recovery with DDU and ECU using FST, $K=$ $20, M_{R}=1$, and $C_{l}=5($ rank=5).

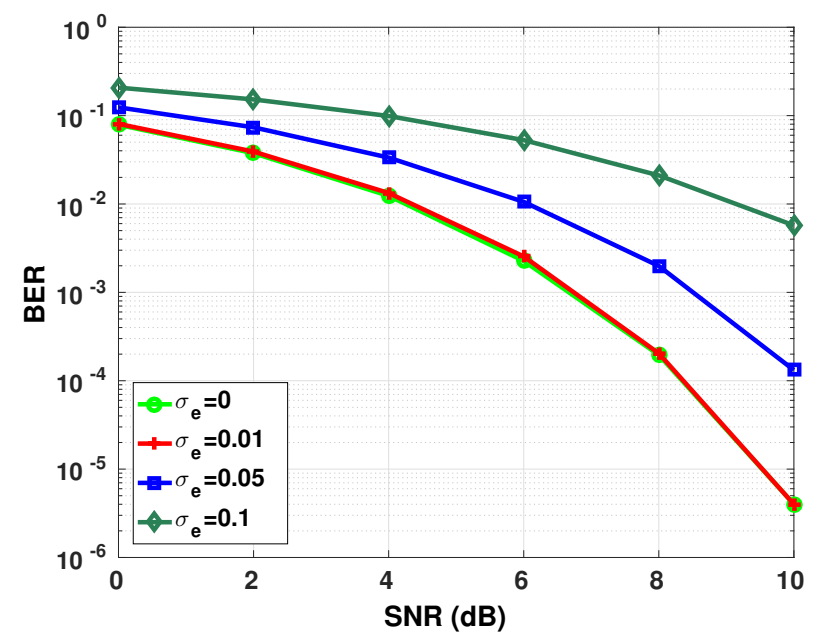

Fig. 13. Average performance for the MRT precoding with estimated channel error, 10 UEs, $M_{R}=1$, and $C_{l}=10($ rank=10).

full channel feedback is equal to $2.5 \mathrm{~dB}$ and $17.5 \mathrm{~dB}$ for a BER of $10^{-3}$, respectively.

In Fig. 15, we show the goodput based on the block error rate (BLER) [60], [61] for different undersampling factors. The goodput is given by $S(1-\mathrm{BLER})$. Assuming that there is no channel coding at the transmitter, the channel is constant during a transmission block, and the Gaussian white noise, the BLER can be expressed as a function of the BER of Fig. 14 following the formulation in [62] (c.f. Chapter 3):

$$
\mathrm{BLER}=1-(1-\mathrm{BER})^{S}
$$

where $S$ is the number of transmitted symbols. The symbols are mapped into a BPSK constellation, and $S=100$ symbols per frame are assumed. At $m=0.1$, the goodput is almost zero due to high BLER. At $m=0.2$, we can achieve full goodput only with $\mathrm{SNR}=10 \mathrm{~dB}$. Furthermore, when $m=0.3$, the performance results are as that of full feedback channel $m=1$.

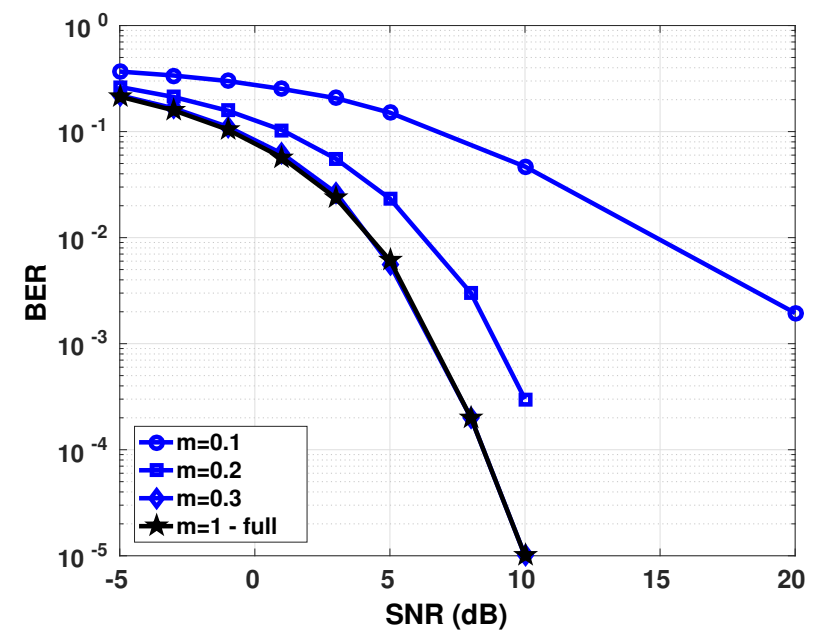

Fig. 14. Average BER performance for the channel recovery with the ECU, $K=10, M_{R}=1$, and $C_{l}=10($ rank=10).

As we can see in Fig. 14, $m=0.3$ is enough to achieve full performance. Thus, since Fig. 14 is related to the goodput of Fig. 15, we conclude that in this scenario, the proposed CSI reconstruction method achieves the ideal goodput performance with only $m=0.3$, which is a remarkable result.

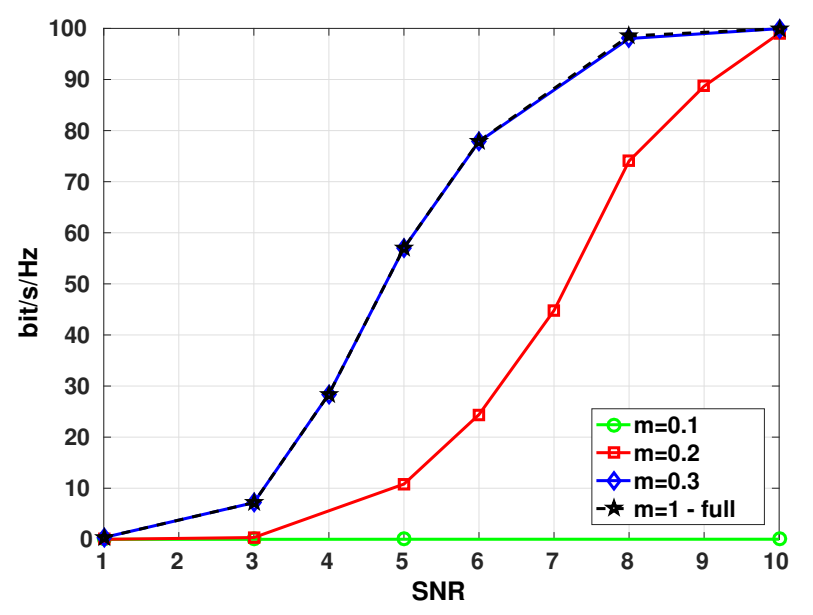

Fig. 15. Comparison for the goodput, $10 \mathrm{UEs}, M_{R}=1$, and $C_{l}=10$ (rank=10).

Considering the Fig. 13 and Fig. 14, we evaluate the proposed framework with ECU, under imperfect CSI, applying MRT with $m \in\{0.1,0.2,0.3,0.4\}$. The results are shown in Fig. 16 and compared to the perfect CSI $\left(\sigma_{e}=0\right)$. With $m=0.3$, the BER performance is the same as the estimated channel error $\sigma_{e}=0.01$. Thereby, even under imperfect CSI, $70 \%$ of feedback data is saved, and it ensures full BER performance. When $\sigma_{e}=0.05$, the estimation error is increased, and thereby, the performance loss is about $2 \mathrm{~dB}$. For $m=0.4$, the loss of performance is around $1 \mathrm{~dB}$.

For $m=0.2$, the average BER performance has the same loss as in Fig. 13 until $\sigma_{e}=0.01$. When the error of estimation is increased to $\sigma_{e}=0.05$, the loss of performance is around 
$3 \mathrm{~dB}$. Thereby, $m=0.2$ is enough to the average BER performance be close to the case on full-feedback as showed the Fig. 13.

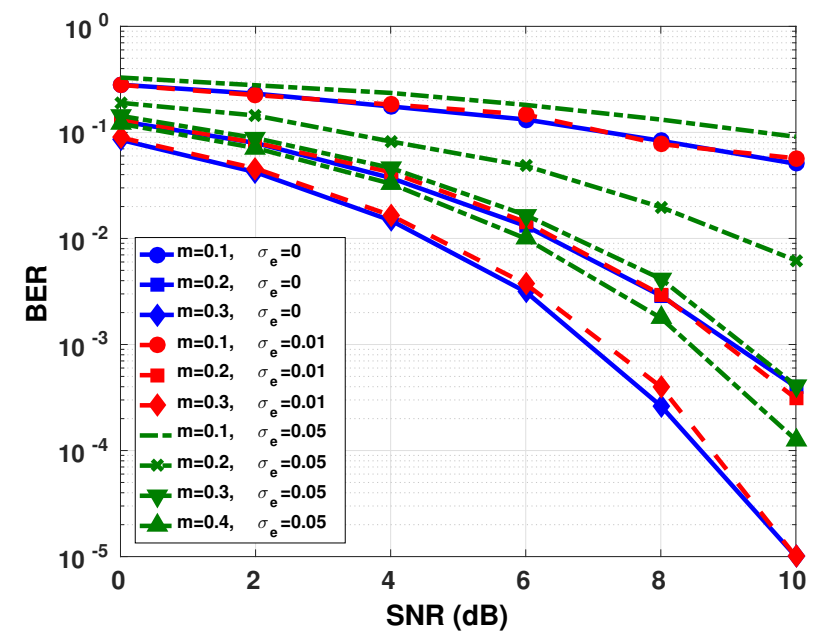

Fig. 16. Average performance for the BER with estimated channel error, $K=10, M_{R}=1$, and $C_{l}=10($ rank=10).

\section{CONCLUSION}

We have provided results suggesting that matrix completion arises as an efficient solution to channel feedback and reconstruction in FDD-based massive MIMO systems to reduce the amount of feedback information to be sent to the BS whenever the MIMO modeled as a low-rank matrix. Our numerical results have shown that matrix completion achieves an accurate recovery of the downlink channel with a small feedback overhead. Consequently, the proposed scheme copes with limitedcapacity uplink feedback channels. Due to undersampling at the UEs, energy consumption can be reduced to lower levels compared with the conventional full feedback. These benefits are possible thanks to the low-rank nature of the channel which happens in a massive MIMO scenario characterized by finite scattering propagation.

As a future work, we intend to evaluate our proposal with high order structures using a tensor model [63], [64]. For instance, assuming a multi-carrier modulation, the subcarrier channels can be concatenated to form a third-order tensor structure [65]. In this case, a tensor completion algorithm [66] can be applied to reconstruct the channel tensor

\section{APPENDIX}

INCOHERENCE PROPERTY OF MASSIVE MIMO CHANNEL

The Assumptions 1 and 2 on the incoherence property of $\mathbf{H}$ when $r=P=1$, can be measured by the singular vector. Comparing (13) with the SVD of $\mathbf{H}=\sum_{i=1}^{P} \sigma_{i} \mathbf{u}_{i} \mathbf{v}_{i}^{H}$, the $\mathbf{u}_{1}=\mathbf{a}_{r}(\theta)$ and $\mathbf{v}_{1}=\mathbf{a}_{t}(\delta)$ are the singular vectors. Thus, all entries of $\mathbf{P}_{U}$ have the same modulus $1 / M_{R}$ and those of $\mathbf{P}_{V}$ have the same modulus $1 / M_{T}$. When $a=a^{\prime}$ and $b=b^{\prime}$,

$$
\begin{array}{r}
\left\langle\mathbf{e}_{a}, \mathbf{P}_{U} \mathbf{e}_{a^{\prime}}\right\rangle=\left[\mathbf{P}_{U}\right]_{a, a^{\prime}}=\frac{1}{M_{R}}, \\
\left|\left\langle\mathbf{e}_{a}, \mathbf{P}_{U} \mathbf{e}_{a^{\prime}}\right\rangle-\frac{1}{M_{R}}\right|=0, \\
\left\langle\mathbf{e}_{b}, \mathbf{P}_{V} \mathbf{e}_{b^{\prime}}\right\rangle=\left[\mathbf{P}_{V}\right]_{b, b^{\prime}}=\frac{1}{M_{T}} \\
\left|\left\langle\mathbf{e}_{b}, \mathbf{P}_{V} \mathbf{e}_{b^{\prime}}\right\rangle-\frac{1}{M_{T}}\right|=0 .
\end{array}
$$

When $a \neq a^{\prime}$ and $b \neq b^{\prime}$,

$$
\begin{gathered}
\left|\left\langle\mathbf{e}_{a}, \mathbf{P}_{U} \mathbf{e}_{a^{\prime}}\right\rangle\right|=\left|\left[\mathbf{P}_{U}\right]_{a, a}\right|=\frac{1}{M_{R}}, \\
\left|\left\langle\mathbf{e}_{b}, \mathbf{P}_{V} \mathbf{e}_{b^{\prime}}\right\rangle\right|=\left|\left[\mathbf{P}_{V}\right]_{b, b}\right|=\frac{1}{M_{T}} .
\end{gathered}
$$

Thus, the Assumptions 1 and 2 with $\mu=1$ are satisfied.

For $r=P \geq 2$, and a sufficiently large number of antennas, the singular vectors of the channel $\mathbf{H}$ converge to the steering vectors [12] (see [67] for a detailed proof). Thereby, all entries of the left and right singular vectors have the same modulus $1 / \sqrt{M_{R}}$ and $1 / \sqrt{M_{T}}$, respectively. Hence, $a=a^{\prime}$ and $b=b^{\prime}$

$$
\begin{aligned}
\left\langle\mathbf{e}_{a}, \mathbf{P}_{U} \mathbf{e}_{a^{\prime}}\right\rangle & =\left[\mathbf{P}_{U}\right]_{a, a}=\frac{P}{M_{R}}, \\
\left\langle\mathbf{e}_{b}, \mathbf{P}_{V} \mathbf{e}_{b^{\prime}}\right\rangle & =\left[\mathbf{P}_{U}\right]_{b, b}=\frac{P}{M_{T}},
\end{aligned}
$$

and, for $a \neq a^{\prime}$ and $b \neq b^{\prime}$,

$$
\begin{aligned}
\left|\left\langle\mathbf{e}_{a}, \mathbf{P}_{U} \mathbf{e}_{a^{\prime}}\right\rangle\right|=\left[\mathbf{P}_{U}\right]_{a, a^{\prime}} & =\left|\sum_{i=1}^{P} u_{i, a} u_{i, a^{\prime}}^{*}\right| \\
& \leq \sum_{i=1}^{P}\left|u_{i, a}\right|\left|u_{i, a^{\prime}}^{*}\right|=\frac{P}{M_{R}}, \\
\left|\left\langle\mathbf{e}_{b}, \mathbf{P}_{V} \mathbf{e}_{b^{\prime}}\right\rangle\right|=\left[\mathbf{P}_{V}\right]_{b, b^{\prime}} & =\left|\sum_{i=1}^{P} v_{i, b} v_{i, b^{\prime}}^{*}\right| \\
& \leq \sum_{i=1}^{P}\left|v_{i, b}\right|\left|v_{i, b^{\prime}}^{*}\right|=\frac{P}{M_{T}} .
\end{aligned}
$$

Thus, the equality $\mu=\sqrt{P}$ satisfies the Assumptions 1 and 2 when $M_{T}, M_{R}$ are very large. This means that when $\mathbf{H}$ is large, it obeys the strong incoherence property with $\mu \approx \sqrt{P}$, and can be recovered with a small number of samples.

\section{REFERENCES}

[1] E. G. Larsson, O. Edfors, F. Tufvesson, and T. L. Marzetta, "Massive MIMO for next generation wireless systems," IEEE Communications Magazine, vol. 52, no. 2, pp. 186-195, 2014. DOI: 10.1109/MCOM.2014.6736761.

[2] T. L. Marzetta, "Noncooperative cellular wireless with unlimited numbers of base station antennas," IEEE Transactions on Wireless Communications, vol. 9, no. 11, pp. 3590-3600, 2010. DOI: 10.1109/TWC.2010.092810.091092.

[3] N. Jindal, "MIMO broadcast channels with finite-rate feedback," IEEE Transactions on Information Theory, vol. 52, no. 11 , pp. 5045-5060, 2006. DOI: 10.1109/TIT.2006.883550. 
[4] W. Santipach and M. L. Honig, "Asymptotic performance of MIMO wireless channels with limited feedback," in IEEE Military Communications Conference, 2003. MILCOM 2003., vol. 1, 2003, 141-146 Vol.1. DOI: 10.1109/MILCOM.2003. 1290092.

[5] C. K. Au-yeung and D. J. Love, "On the performance of random vector quantization limited feedback beamforming in a MISO system," IEEE Transactions on Wireless Communications, vol. 6, no. 2, pp. 458-462, 2007. DOI: 10.1109/TWC. 2007.05351.

[6] D. J. Love, R. W. Heath, V. K. N. Lau, D. Gesbert, B. D. Rao, and M. Andrews, "An overview of limited feedback in wireless communication systems," IEEE Journal on Selected Areas in Communications, vol. 26, no. 8, pp. 1341-1365, 2008. DOI: 10.1109/JSAC.2008.081002.

[7] X. Rao, V. K. N. Lau, and X. Kong, "CSIT estimation and feedback for FDD multi-user massive MIMO systems," in 2014 IEEE International Conference on Acoustics, Speech and Signal Processing (ICASSP), 2014, pp. 3157-3161. DOI: 10. 1109/ICASSP.2014.6854182.

[8] P. H. Kuo, H. T. Kung, and P. A. Ting, "Compressive sensing based channel feedback protocols for spatially-correlated massive antenna arrays," in 2012 IEEE Wireless Communications and Networking Conference (WCNC), 2012, pp. 492-497. DOI: 10.1109/WCNC.2012.6214417.

[9] J. C. Shen, J. Zhang, K. C. Chen, and K. B. Letaief, "Highdimensional CSI acquisition in massive MIMO: Sparsityinspired approaches," Computing Research Repository CoRR, vol. abs/1505.00426, 2015.

[10] M. S. Sim, J. Park, C. Chae, and R. W. H. Jr., "Compressed channel feedback for correlated massive MIMO systems," Computing Research Repository CoRR, vol. abs/1503.09002, 2015.

[11] H. Wang, W. Wang, and Z. Zhang, "On the design of hybrid limited feedback for massive MIMO systems," in 2014 IEEE International Conference on Communications (ICC), 2014 pp. 4795-4800. DOI: 10.1109/ICC.2014.6884079.

[12] R. Hu, J. Tong, J. Xi, Q. Guo, and Y. Yu, "Robust channel estimation for switch-based mmwave MIMO systems," in Proc. 9th Int. Conf. Wireless Communications and Signal Processing (WCSP), Oct. 2017, pp. 1-7. DOI: 10.1109/WCSP. 2017.8170914.

[13] B. Lee, J. Choi, J. y. Seol, D. J. Love, and B. Shim, "Antenna grouping based feedback reduction for FDD-based massive MIMO systems," in 2014 IEEE International Conference on Communications (ICC), 2014, pp. 4477-4482. DOI: 10.1109/ ICC.2014.6884026.

[14] G. Wunder and J. Schreck, "Feedback information transmission and scheduling in a radio access network," Patent EP 2528290 A1, May 2011.

[15] S. Sun, A. P. Petropulu, and W. U. Bajwa, "Target estimation in colocated MIMO radar via matrix completion," in 2013 IEEE International Conference on Acoustics, Speech and Signal Processing, 2013, pp. 4144-4148. DOI: 10.1109/ICASSP.2013. 6638439.

[16] W. Shen, L. Dai, B. Shim, S. Mumtaz, and Z. Wang, "Joint CSIT acquisition based on low-rank matrix completion for FDD massive MIMO systems," IEEE Communications Letters, vol. 19 , no. 12, pp. 2178-2181, 2015. DOI: 10.1109/LCOMM. 2015.2492960.

[17] M. D. Migliore, D. Pinchera, M. Lucido, F. Schettino, and G. Panariello, "MIMO channel-state estimation in the presence of partial data and/or intermittent measurements," Electronics, vol. 6, no. 2, 2017. DOI: 10.3390/electronics6020033.

[18] T. K. Y. Lo, "Maximum ratio transmission," IEEE Transactions on Communications, vol. 47, no. 10, pp. 1458-1461, 1999. DOI: $10.1109 / 26.795811$.

[19] F. Rusek, D. Persson, B. K. Lau, E. G. Larsson, T. L. Marzetta, O. Edfors, and F. Tufvesson, "Scaling up MIMO
Opportunities and challenges with very large arrays," IEEE Signal Processing Magazine, vol. 30, no. 1, pp. 40-60, 2013. DOI: 10.1109/MSP.2011.2178495.

[20] E. J. Candès and B. Recht, "Exact low-rank matrix completion via convex optimization," in 46th Annual Allerton Conference on Communication, Control, and Computing, 2008, pp. 806812. DOI: 10.1109/ALLERTON.2008.4797640.

[21] E. J. Candès and Y. Plan, "Matrix completion with noise," Proceedings of the IEEE, vol. 98, no. 6, pp. 925-936, 2010. DOI: 10.1109/JPROC.2009.2035722.

[22] M. A. Davenport and J. Romberg, "An overview of low-rank matrix recovery from incomplete observations," IEEE Journal of Selected Topics in Signal Processing, vol. 10, no. 4, pp. 608 622, 2016. DOI: 10.1109/JSTSP.2016.2539100.

[23] J. V. Leeuwen, Handbook of Theoretical Computer Science: Algorithms and Complexity, Warwick, A. R. Meyer, and M. Nival, Eds. Cambridge, MA, USA: MIT Press, 1990.

[24] M. Fazel, H. Hindi, and S. P. Boyd, "Log-det heuristic for matrix rank minimization with applications to hankel and euclidean distance matrices," in Proceedings of the American Control Conference, vol. 3, 2003, pp. 2156-2162. DOI: 10. 1109/acc.2003.1243393.

[25] B. Recht, "A simpler approach to matrix completion," J. Mach. Learn. Res., vol. 12, pp. 3413-3430, Dec. 2011.

[26] M. Grant and S. Boyd, Cvx: Matlab software for disciplined convex programming, version 2.1 , http://cvxr.com/cvx, Mar. 2014.

[27] J.-. F. Cai, E. J. Candès, and Z. Shen, "A singular value thresholding algorithm for matrix completion," SIAM J. on Optimization, vol. 20, no. 4, pp. 1956-1982, Mar. 2010. DOI: $10.1137 / 080738970$

[28] W. Dai, O. Milenkovic, and E. Kerman, "Subspace evolution and transfer (SET) for low-rank matrix completion," Computing Research Repository CoRR, vol. abs/1006.2195, 2010.

[29] R. H. Keshavan, S. Oh, and A. Montanari, "Matrix completion from a few entries," in The IEEE International Symposium on Information Theory, (ISIT'09), 2009, pp. 324-328. DOI: 10.1109/ISIT.2009.5205567.

[30] L. Balzano, R. D. Nowak, and B. Recht, "Online identification and tracking of subspaces from highly incomplete information," Computing Research Repository CoRR, vol. abs/1006.4046, 2010.

[31] M. Fornasier, H. Rauhut, and R. Ward, "Low-rank matrix recovery via iteratively reweighted least squares minimization," SIAM J. on Optimization, vol. 21, no. 4, pp. 1614-1640, Dec. 2011. DOI: $10.1137 / 100811404$.

[32] A. Majumdar and R. K. Ward, "Some empirical advances in matrix completion," Signal Processing, vol. 91, no. 5, pp. 1334 -1338, 2011. DOI: http://dx.doi.org/10.1016/j.sigpro.2010.12. 005 .

[33] E. J. Candès and T. Tao, "The power of convex relaxation: Near-optimal matrix completion," IEEE Transactions on Information Theory, vol. 56, no. 5, pp. 2053-2080, 2010. DOI: 10.1109/TIT.2010.2044061.

[34] J.-A. Tsai, R. M. Buehrer, and B. D. Woerner, "The impact of AOA energy distribution on the spatial fading correlation of linear antenna array," in Vehicular Technology Conference. IEEE 55th Vehicular Technology Conference. VTC Spring 2002 (Cat. No.02CH37367), vol. 2, 2002, 933-937 vol.2. DOI: 10.1109/VTC.2002.1002625.

[35] G. H. Song, J. Brady, and A. Sayeed, "Beamspace MIMO transceivers for low-complexity and near-optimal communication at mm-wave frequencies," in 2013 IEEE International Conference on Acoustics, Speech and Signal Processing, 2013, pp. 4394-4398. DOI: 10.1109/ICASSP.2013.6638490.

[36] T. S. Rappaport, S. Sun, R. Mayzus, H. Zhao, Y. Azar, K. Wang, G. N. Wong, J. K. Schulz, M. Samimi, and F. Gutierrez, "Millimeter wave mobile communications for $5 \mathrm{G}$ cellular: It 
will work!" IEEE Access, vol. 1, pp. 335-349, 2013. DOI: 10.1109/ACCESS.2013.2260813.

[37] H. Xie, F. Gao, and S. Jin, "An overview of low-rank channel estimation for massive MIMO systems," IEEE Access, vol. 4, pp. 7313-7321, 2016. DOI: 10.1109/ACCESS.2016.2623772.

[38] Z. Zhou, J. Fang, L. Yang, H. Li, Z. Chen, and S. Li, "Channel estimation for millimeter-wave multiuser MIMO systems via parafac decomposition," IEEE Transactions on Wireless Communications, vol. 15, no. 11, pp. 7501-7516, Nov. 2016. DOI: 10.1109/TWC.2016.2604259.

[39] Z. Gao, L. Dai, Z. Wang, and S. Chen, "Spatially common sparsity based adaptive channel estimation and feedback for FDD massive MIMO," IEEE Transactions on Signal Processing, vol. 63, no. 23, pp. 6169-6183, Dec. 2015. DOI: 10.1109/ TSP.2015.2463260.

[40] A. Adhikary, J. Nam, J. Y. Ahn, and G. Caire, "Joint spatial division and multiplexing - the large-scale array regime," IEEE Transaction Information Theory, vol. 59, no. 10, pp. 64416463, 2013. DOI: 10.1109/TIT.2013.2269476.

[41] J. Nam, A. Adhikary, J. Y. Ahn, and G. Caire, "Joint spatial division and multiplexing: Opportunistic beamforming, user grouping and simplified downlink scheduling," IEEE Journal of Selected Topics in Signal Processing, vol. 8, no. 5, pp. 876890, 2014. DOI: 10.1109/JSTSP.2014.2313808.

[42] A. Sayeed and J. Brady, "Beamspace MIMO for highdimensional multiuser communication at millimeter-wave frequencies," in 2013 IEEE Global Communications Conference (GLOBECOM), 2013, pp. 3679-3684. DOI: 10.1109/ GLOCOM.2013.6831645.

[43] J. Hogan and A. Sayeed, "Beam selection for performancecomplexity optimization in high-dimensional MIMO systems," in 2016 Annual Conference on Information Science and Systems (CISS), 2016, pp. 337-342. DOI: 10.1109/CISS.2016. 7460525 .

[44] M. K. Samimi and T. S. Rappaport, "Local multipath model parameters for generating $5 \mathrm{G}$ millimeter-wave 3GPP-like channel impulse response," in Proc. 10th European Conf. Antennas and Propagation (EuCAP), Apr. 2016, pp. 1-5. DOI: 10.1109/EuCAP.2016.7481410.

[45] E. Björnson, J. Hoydis, L. Sanguinetti, et al., "Massive mimo networks: Spectral, energy, and hardware efficiency," Foundations and Trends ${ }^{\circledR}$ in Signal Processing, vol. 11, no. 3-4, pp. 154-655, 2017.

[46] S. Buzzi and C. D'Andrea, "Massive MIMO 5G cellular networks: Mm-wave vs. micro-wave frequencies.," Feb. 23, 2017. arXiv: 1702.07187v1 [CS.IT].

[47] H. Xie, F. Gao, S. Zhang, and S. Jin, "A unified transmission strategy for TDD/FDD massive MIMO systems with spatial basis expansion model," IEEE Transactions on Vehicular Technology, vol. 66, no. 4, pp. 3170-3184, Apr. 2017. DOI: 10. 1109/TVT.2016.2594706.

[48] B. Eriksson, L. Balzano, and R. D. Nowak, "High-rank matrix completion and subspace clustering with missing data," CoRR, vol. abs/1112.5629, 2011. arXiv: 1112.5629.

[49] E. Dahlman, S. Parkvall, and J. Skold, 4G, LTE-Advanced Pro and The Road to 5G, Third Edition, 3rd. Academic Press, 2016.

[50] M. Coldrey, U. Engström, K. W. Helmersson, M. Hashemi, L. Manholm, and P. Wallentin, "Wireless backhaul in future heterogeneous networks," Ericsson Review, vol. 91, pp. 1-11, 2014.

[51] S. Hur, T. Kim, D. J. Love, J. V. Krogmeier, T. A. Thomas, and A. Ghosh, "Multilevel millimeter wave beamforming for wireless backhaul," in 2011 IEEE GLOBECOM Workshops (GC Wkshps), 2011, pp. 253-257. DOI: 10.1109/GLOCOMW. 2011.6162448.

[52] - "Millimeter wave beamforming for wireless backhaul and access in small cell networks," IEEE Transactions on
Communications, vol. 61, no. 10, pp. 4391-4403, 2013. DOI: 10.1109/TCOMM.2013.090513.120848.

[53] A. Swindlehurst, E. Ayanoglu, P. Heydari, and F. Capolino, "Millimeter-wave massive MIMO: The next wireless revolution?" Communications Magazine, IEEE, vol. 52, no. 9, pp. 56-62, 2014. DOI: 10.1109/MCOM.2014.6894453.

[54] E. Castañeda, A. Silva, A. Gameiro, and M. Kountouris, "An overview on resource allocation techniques for multi-user MIMO systems," IEEE Communications Surveys Tutorials, vol. 19, no. 1, pp. 239-284, 2017. DOI: 10.1109/COMST. 2016.2618870

[55] A. Adhikary, E. A. Safadi, M. K. Samimi, R. Wang, G. Caire, T. S. Rappaport, and A. F. Molisch, "Joint spatial division and multiplexing for mm-wave channels," IEEE Journal on Selected Areas in Communications, vol. 32, no. 6, pp. 12391255, 2014. DOI: 10.1109/JSAC.2014.2328173.

[56] H. Yin, D. Gesbert, M. Filippou, and Y. Liu, "A coordinated approach to channel estimation in large-scale multiple-antenna systems," IEEE Journal on Selected Areas in Communications, vol. 31, no. 2, pp. 264-273, 2013. DOI: 10.1109/JSAC.2013. 130214.

[57] R. H. Keshavan, A. Montanari, and S. Oh, "Matrix completion from noisy entries," Journal of Machine Learning Research, vol. 99, pp. 2057-2078, Jun. 11, 2009. arXiv: 0906.2027v2 [CS.LG]

[58] S. Haykin, Adaptive Filter Theory (3rd Ed.) Upper Saddle River, NJ, USA: Prentice-Hall, Inc., 1996.

[59] A. Sobral, T. Bouwmans, and E.-h. Zahzah, "Lrslibrary: Lowrank and sparse tools for background modeling and subtraction in videos," in Robust Low-Rank and Sparse Matrix Decomposition: Applications in Image and Video Processing, CRC Press, Taylor and Francis Group., 2015.

[60] W. C. Freitas, F. R. P. Cavalcanti, A. L. F. de Almeida, and R. R. Lopes, "Exploiting dimensions of the MIMO wireless channel: Multidimensional link adaptation," in 2005 IEEE 61st Vehicular Technology Conference, vol. 2, 2005, 924-928 Vol. 2. DOI: 10.1109/VETECS.2005.1543441.

[61] D. Cavalcante and C. C. Cavalcante, "Channel estimation in link adaptation strategies for MIMO-OFDM systems," in World Wireless Research Forum, 2006.

[62] D. Bartolomé, "Fairness analysis of wireless beamforming schedulers," PhD thesis, Universitat Politécnica de Catalunya, 2005

[63] D. Nion and N. Sidiropoulos, "Tensor algebra and multidimensional harmonic retrieval in signal processing for MIMO radar," Signal Processing, IEEE Transactions on, vol. 58, no. 11, pp. 5693-5705, 2010. DOI: 10.1109 / TSP. 2010 . 2058802.

[64] L.-T. Huang, A. L. de Almeida, and H. So, "Target estimation in bistatic MIMO radar via tensor completion," Signal Process., vol. 120, no. C, pp. 654-659, Mar. 2016. DoI: 10.1016/ j.sigpro.2015.09.036.

[65] A. L. F. de Almeida, G. Favier, P. C. L. Costa, and J. C. M. Mota, "Overview of tensor decompositions with applications to communications," in Signals and Images: Advances and Results in Speech, Estimation, Compression, Recognition, Filtering, and Processing, Chapter 12, R. Coelho, V. Nascimento, R. de Queiroz, J. Romano, and C. Cavalcante, Eds., CRCPress, Jan. 2016, pp. 325-356.

[66] M. Signoretto, R. Van de Plas, B. De Moor, and J. Suykens, "Tensor versus matrix completion: A comparison with application to spectral data," Signal Processing Letters, IEEE, vol. 18, no. 7 , pp. 403-406, 2011. DOI: 10.1109/LSP.2011.2151856.

[67] O. E. Ayach, R. W. Heath, S. Abu-Surra, S. Rajagopal, and $\mathrm{Z}$. Pi, "The capacity optimality of beam steering in large millimeter wave MIMO systems," in Proc. IEEE 13th Int. Workshop Signal Processing Advances in Wireless Communications (SPAWC), Jun. 2012, pp. 100-104. DOI: 10.1109/ SPAWC.2012.6292865 


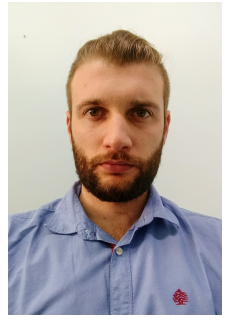

Samuel Tumelero Valduga received the B.S.E.E and M.Sc in electrical engineering and computer science from Federal University of Santa Maria, Santa Maria, RS, Brazil, in 2012 and 2014, respectively and Ph.D. degree in Teleinformatics Engineering from the Federal University of Ceará, Fortaleza, Brazil, in 2018 .

$\mathrm{He}$ is currently an Adjunct Professor with the Department of Electronics and Computing (DELC) with Telecommunications Engineering of the Federa University of Santa Maria. He is member of Wireless Telecommunications Research Group (GTEL), Fortaleza, Brazil. From 2016 to 2017, he held a one year research position at the I3S Laboratory, CNRS, France. His research interests are Massive MIMO, Matrix and Tensor Completion, Pilot Contamination, Beamforming, Precoding, and Channel Estimation.

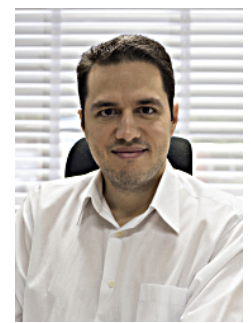

André L. F. de Almeida received the double Ph.D. degree in Sciences and Teleinformatics Engineering from the University of Nice, Sophia Antipolis, France, and the Federal University of Ceará, Fortaleza, Brazil, in 2007.

$\mathrm{He}$ is currently an Assistant Professor with the Department of Teleinformatics Engineering of the Federal University of Ceará. During fall 2002, he was a visiting researcher at Ericsson Research Labs, Stockholm, Sweden. From 2007 to 2008, he held a one year research position at the I3S Laboratory, CNRS, France. In 2008, he was awarded a CAPES/COFECUB research fellowship with the I3S Laboratory, CNRS, France. He currently holds a productivity research fellowship from CNPq (the Brazilian National Council for Scientific and Technological Development). In 2012, 2013 and 2015, he was a Visiting Professor at the University of Nice Sophia Antipolis, France. He served as an Associate Editor of the IEEE Transactions on Signal Processing (2012-1016). He is currently an Associate Editor of the IEEE Signal Processing Letters and serves as a general co-chair of IEEE CAMSAP2017. His research interests lie in the areas of signal processing for communications and sensor array processing, and include blind methods for channel identification, equalization and source separation, MIMO receivers, multilinear algebra and tensor-based signal processing applied to communications and data analysis

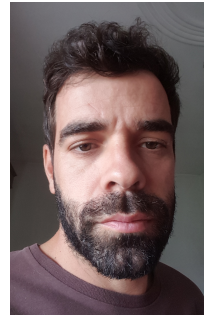

Carlos Filipe Moreira e Silva received a five years diploma and M.Sc. degrees in Electronics and Telecommunications Engineering from the University of Aveiro (UA), Portugal, in 2005 and 2010, respectively. In 2015 he also received a Ph.D. degree in Teleinformatics Engineering from the Federal University of Ceará, Brazil

Since 2006, Carlos Silva has been a researcher in several European projects, such as WINNER II (system requirements for beyond 3rd generation wireless networks), FUTON (RRM for wireless and optical networks), and COGEU (cognitive radio systems for efficient use and sharing of TVWS in the European context). Currently, he has a postdoc position at the GTEL, Brazil, where he manages GTEL's team in the European-Brazilian project FUTEBOL and also works in cooperation projects with Ericsson Research. His main research interests include: spectrum usage optimization, scarcity and management, namely related with TV White Spaces (TVWS), Licensed Shared Access (LSA), Internet of Things (IoT), and Device-toDevice (D2D) communications; testbed experimentation; and architectural aspects of the future $5 \mathrm{G}$ networks.

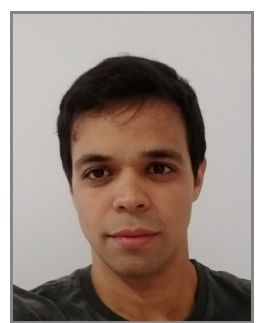

Daniel C. Araujo received his Bachelor degree in Telecommunications Engineering from Fortaleza University (UNIFOR) in 2010 and his M.S. and $\mathrm{PhD}$ degrees in Teleinformatics Engineering from Federal University of Ceará, Brazil in 2012 and 2016, respectively

During his studies, he was supported by the Brazilian agency CAPES and Ericsson. During his $\mathrm{PhD}$, he was visiting researcher at ERICSSON in 2013 and 2015 up to 2016 . He is currently a post-doc researcher of GTEL. His areas of interests concern to channel estimation, sparse signal processing, massive MIMO and Hybrid beamforming.

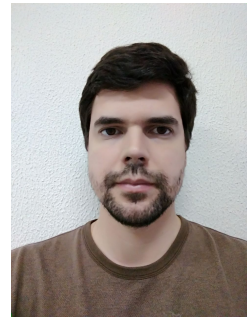

Igor Moáco Guerreiro received the B.S., M.S. and Ph.D. degrees in Teleinformatics Engineering from the Federal University of Ceará (UFC), Brazil, in 2007, 2010 and 2016, respectively.

He currently holds a post-doc position at the UFC Department of Teleinformatics Engineering. Since 2007 he has been a researcher at Wireless GTEL, Brazil, working in research projects within a technical cooperation with Ericsson Research, Sweden. In 2008, he was a guest researcher at Virginia Tech Advanced Research Institute (ARI), Arlington, Virginia, USA. Before starting the Ph.D. course at UFC, he visited Ericsson in 2010 in Luleå, Sweden, for 5 months, and another time in San José, California, USA, for 3 months. As a Ph.D. student, he visited both Ericsson and the Royal Institute of Technology (KTH) in 2014-15 in Stockholm, Sweden, for a year-long period. Some topics of his research interests include techniques for MIMO transceiver design, strategies for distributed optimization for wireles communication systems, modeling and simulation of cellular communication, and dynamic spectrum access methodologies. 